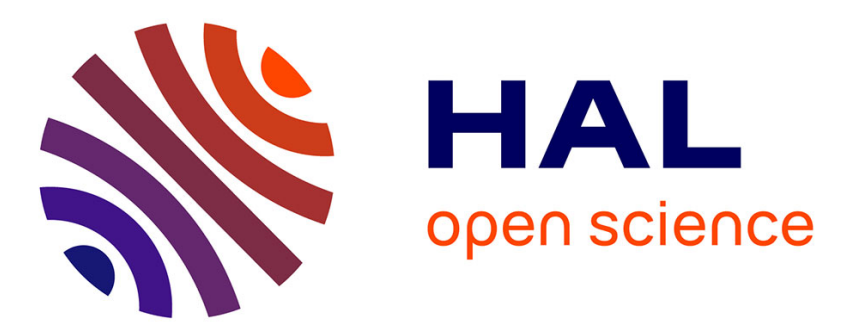

\title{
Recent advances in Surface Plasmon Resonance for biosensing applications and future prospects
}

\author{
Biplob Mondal, Shuwen Zeng
}

\section{To cite this version:}

Biplob Mondal, Shuwen Zeng. Recent advances in Surface Plasmon Resonance for biosensing applications and future prospects. Nanophotonics in Biomedical Engineering, 2020. hal-02915657

\section{HAL Id: hal-02915657 \\ https://hal-unilim.archives-ouvertes.fr/hal-02915657}

Submitted on 14 Aug 2020

HAL is a multi-disciplinary open access archive for the deposit and dissemination of scientific research documents, whether they are published or not. The documents may come from teaching and research institutions in France or abroad, or from public or private research centers.
L'archive ouverte pluridisciplinaire $\mathbf{H A L}$, est destinée au dépôt et à la diffusion de documents scientifiques de niveau recherche, publiés ou non, émanant des établissements d'enseignement et de recherche français ou étrangers, des laboratoires publics ou privés. 


\title{
Recent advances in Surface Plasmon Resonance for biosensing applications and future prospects
}

\author{
Biplob Mondal ${ }^{1}$, Shuwen Zeng ${ }^{2 *}$
}

1. Department of Electronics and Communication Engineering, Tezpur University, Tezpur 784028, Assam, India

2. XLIM Research Institute, UMR 7252 CNRS/University of Limoges, 123, Avenue Albert Thomas, 87060 Limoges CEDEX, France

* Correspondence: shuwen.zeng@unilim.fr

\section{Introduction}

Optical biosensors have been widely applied among the scientific community for different aspects, most commonly in monitoring protein-protein or nucleic acid hybridization interactions and association and determining dissociation kinetics, etc. The application of Surface Plasmon is recognized widely in recent years as a potential tool for fast screening of bioanalytes where the current main challenge is the detection of nanomolar and picomolar concentration of hard-toidentify biomolecules. Surface Plasmon Resonance (SPR) interrogation is one of the most advanced and unique optical transduction methods for label-free and real-time detecting biological species in molecular level that uses an optical approach to measure a change in the refractive index unit of target sensing media. The sensing medium is in close vicinity of a metallic surface that consisted of a large number of free electrons to interact with the incident light. Plasmon resonance in this phenomenon can result in extraordinary detection limit due to its capability to give extremely high sensitivity towards even a tiny change in refractive index at the sensing interface. This chapter presents a brief review on Kretschmann configuration-based surface plasmon resonance technique for the biosensing areas. Here, we will present the latest trend to design and fabricate portable setup for SPR measurement along with discussions related to target antibody immobilization, choice of metal for improved sensitivity and accuracy of measurement. The chapter also provides the insights on the future trends of SPR technology for superior sensing performance.

\section{History of SPR technology}

In 1902, R.W. Wood observed anomaly spectra of dark and light bands occurred in the reflected light when polarized light is incident on a mirror with diffraction grating at the surface [1]. This appeared to be a remarkable physical phenomenon that took nearly four decade to be physically 
interpreted with the excitation of surface plasmons by attenuated total reflection (ATR) by Otto [2], Raether, and Kretschmann [3] independently in the year of 1968. The fundamental principle of their demonstration were based on the studies made by Zenneck [4] in 1907 theoretically proving the existence of radio frequency surface electromagnetic (EM) waves at the boundary of a metal (lossy) and dielectric (loss-free) medium, Ritchie [5] in 1957 theoretically demonstrating the existence of surface plasma at metal surface, while Swan and Powell's [6] work on the excitation of surface plasmons at metallic interfaces with electrons, and later by Stern and Ferrell [7] to show surface electromagnetic waves at a metallic surface involved the coupling between electromagnetic radiation and surface plasmons.

In its early stage, the unique property of surface plasmon resonance (SPR) being very sensitive to its boundary conditions was utilized in determining the optical properties of cadmium arachidate thin films deposited on metal surface by Pockrand [8] and Wahling [9] independently. In the year of 1982, Nylander [10] have demonstrated the utility of SPR in gas sensing. Later, Liedberg et. al [11] working at the laboratory of applied physics in Linkoping University, Sweden reported biosensing with surface plasmon resonance for the first time. They reported immunosensing of antiIgG (anti-immunoglobulin $\mathrm{G}$ ) down to the concentration of $0.2 \mu \mathrm{g} / \mathrm{ml}$ using $60 \mathrm{~nm}$ thick silver films deposited on microscopic slide. Since than, an increasing number of the SPR approaches is developed in health care research, fundamental biological studies, drug design/screening, clinical diagnosis, food and environmental monitoring, defense security, and agricultural insecticide research [12-14]. Over the years, several generation of instrumentation for SPR bio-sensing has been witnessed from prism and grating coupled [15] instrumentation to fibre coupled SPR sensing for point-of-care application [16-18].

Current trends in SPR technology directed towards improving the sensitivity and accuracy of measurements with the application of multilayer graphene or Molybdenum disulfide $\left(\mathrm{MoS}_{2}\right)$ coating, wear resistance of the sensitive element and developing methods for regeneration of receptors for multiple use [19-21]. SPR imaging with integrated Microfluidics lab-on-a-chip (LOC) for point-of-care (POC) application in medical and clinical theranostics appears to be the promising technology of near future [22].

\section{Fundamental theory and principle of Surface Plasmon Resonance}

Surface plasmon resonance (SPR) is an optical phenomenon that induces a charge density oscillation wave that could propogate along the metal dielectric interface (metal and dielectric layer having opposite sign for their dielectric constants) when excited by p-polarized light. These surface plasmons also referred to as surface plasmon wave (SPW) can only be excited by a longitudinal (TM or p-polarized) electric field (along the direction of light propagation), which would exponentially decay in the hybrid layer of metal and dielectric. The propagation constant of a SPW is defined as [23]: 


$$
K_{s p}=\frac{w}{c} \sqrt{\frac{\varepsilon_{m} \varepsilon_{d}}{\varepsilon_{m}+\varepsilon_{d}}}
$$

Where $c$ is the speed of light in vacuum, $w$ is angular frequency, and $\varepsilon_{m}$ and $\varepsilon_{d}$ are respectively the dielectric constants of metal layer and dielectric layer. Here, the real part of $\varepsilon_{m}$ must be negative, and its absolute value smaller than $\varepsilon_{d}$ to promise the metals could supports SPW. The surface plasmon waves (SPWs) have maximum field intensities at the metal-dielectric interface with limited propagation length, usually $200 \mathrm{~nm}$ to $400 \mathrm{~nm}$. The electromagnetic field decreased exponentially in both the media and the majority of which is concentrated in the dielectric [17] indicating SPW is highly sensitive to the changes in properties of the dielectric.

The propagation constant of a light wave propagating with a frequency $w$ in free space is given by

$$
K_{d}=\frac{w}{c} \sqrt{\varepsilon_{d}}
$$

Based on eq. (1) and eq. (2), the dielectric constants of metal and dielectric being negative and positive respectively infers that the wave propagation constant of SPW should be always higher than that of wave propagating in dielectric. Direct light therefore cannot excite surface plasmon at metal-dielectric interface, and hence to satisfy the condition of resonance the momentum of the incident wave propagating through the dielectric that has to be enhanced. At resonance the wave vector of the incident and surface plasmon wave (SPW) matches with each other leading to a reduced intensity of the reflected light. To date, three methods are popularly used for matching the momentum that uses high refractive index material for momentum matching: Attenuated total reflection (ATR) in prism coupler, diffraction at the surface of diffraction grating, and optical waveguides.

\section{Light wave coupling techniques}

In prism coupling configuration, surface plasmons (SPs) are usually excited with an evanescent wave from a high-refractive-index glass prism under Attenuated Total Reflection (ATR) condition [17]. When p-polarized is passed through a prism coated with metal at its base which remains in contact with dielectric media of low refractive index and $\theta_{\text {inc }}$ is greater than or equal to critical angle for total internal reflection, evanescent wave is generated at the prism metal interface. This facilitates the excitation of surface Plasmons (SPs) which occurs when the wave vector of the propagation constant of incident light through the prism matches with that of the Surface Plasmon with same frequency and polarization state. This occurs at a specific angle of incidence $\theta_{\text {res }}$ and the resonance condition is given as 


$$
\frac{w}{c} \sqrt{\varepsilon_{p}} \sin \theta_{\text {res }}=\frac{w}{c} \sqrt{\left(\frac{\varepsilon_{m} \varepsilon_{d}}{\varepsilon_{m}+\varepsilon_{d}}\right)}
$$

Where the left side of the eq. (3) is the wave propagation constant of the incident light in the direction parallel to the metal surface and $\varepsilon_{p}$ is the refractive index of prism. The propagation constant of evanescent wave and SPW can be matched by controlling the angle of incidence and frequency of the incident beam. This method is schematically depicted in fig. 1(a). The ATR method is conventionally implemented in two different configurations proposed by Otto and Kretschmann. The core of an optical waveguide (for example, optical silica fiber) can be used to replace the bulky prism used in ATR method to get the benefit of simple and flexible design and miniaturized sensor system. Evanescent waves are excited at the core-cladding interface when light is guided through optical fiber. If a portion of the cladding is removed and deposited with thin metal layer, light entering the region can evanescently penetrate through the metal layer exciting SPW at the core-metal interface. The method is depicted in fig. 1(b). It is worth noting that the coupling of the evanescent field with SPs depends strongly on the wavelength of incident light, and the dielectric constants of the fiber/metal layer. Momentum enhancement to compensate the wave vector mismatch between the incident light waves and SPWs can also be achieved with the diffraction of the light at the surface of metallic diffraction grating as shown in figure 1(c). Conventionally a sinusoidal grating is used with which the wave vector enhancement of the diffracted wave proportional to the period of the grating can be achieved [24]. Since fabrication of diffraction grating of given specification is expensive, inexpensive gold-coated diffraction gratings using commercial CD-R type optical storage disk is reported as a low-cost alternative for the fabrication of integrated SPR-based optical sensors [25].

(a)
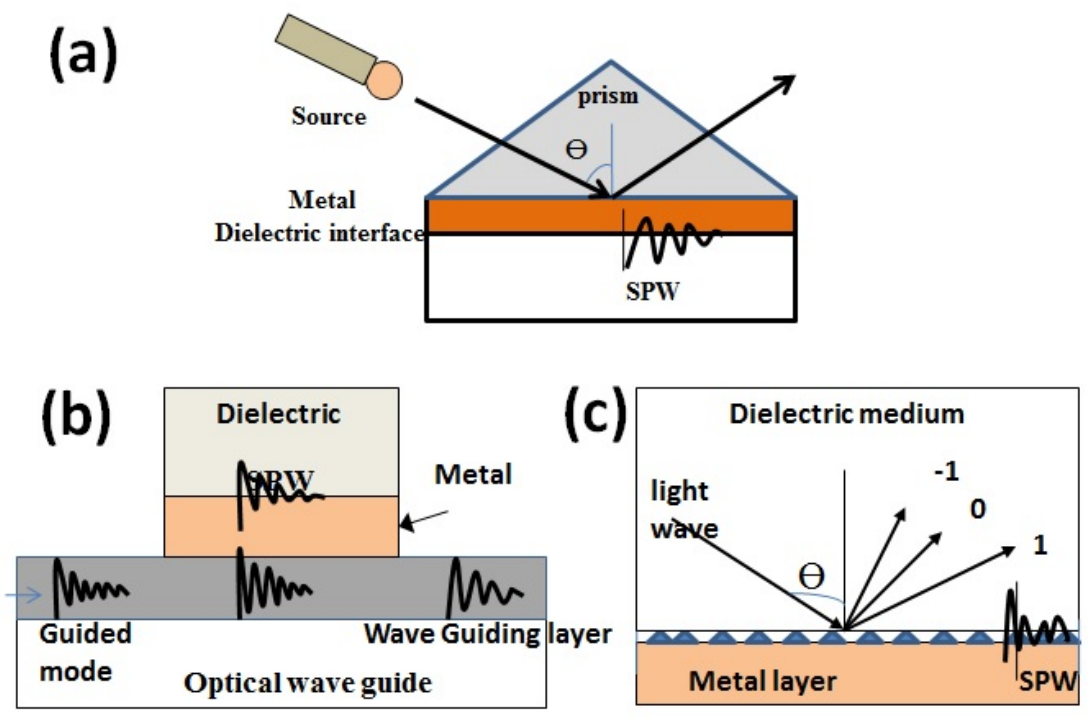
Fig. 1:- Excitation of surface plasmon waves (SPWs) based on: (a) prism coupling (b) optical waveguide (c) diffracting grating

\section{Sensing with Surface plasmon resonance}

The interaction of light wave with SPW results in changes in the optical characteristics like intensity, phase etc., of the reflected light which is correlated with the propagation constant of the SPWs. Accordingly, based on the measured characteristics SPR devices are classified as intensity [28], angular [26], wavelength [27], phase or polarization [29] modulation-based device. Excitation of SPW at metal-dielectric interface results in resonant energy transfer into the SPR form the incident photons reducing the reflected light energy. In the Kretschmann configuration, which is a widely used scheme for SPR sensing, p-polarized light from a light source is directed through a high-refractive-index prism with a thin metal layer $(\sim 50 \mathrm{~nm})$ deposited at the base. If the reflected light intensity is measured in terms of incident angle, a sharp dip in the reflected intensity could be observed at particular angle of incidence due to resonant transfer of energy to the SPW. The angle at which this resonance condition could be fulfilled is called resonance angle. Optimum coupling between the incident light and the surface Plasmon wave (surface plasmon resonance condition) is strongly dependent on the refractive index of the dielectric medium close to the surface of the metal layer. This is attributed to the fact that the electromagnetic field is strongly concentrated in the dielectric. Thus, the SPR technique is highly sensitive and surface specific experimental for small molecule interactions. If one increased the refractive index of the dielectric sensing media, the resonance angle would have a shift to a larger angle [30-32] as shown in figure 2.

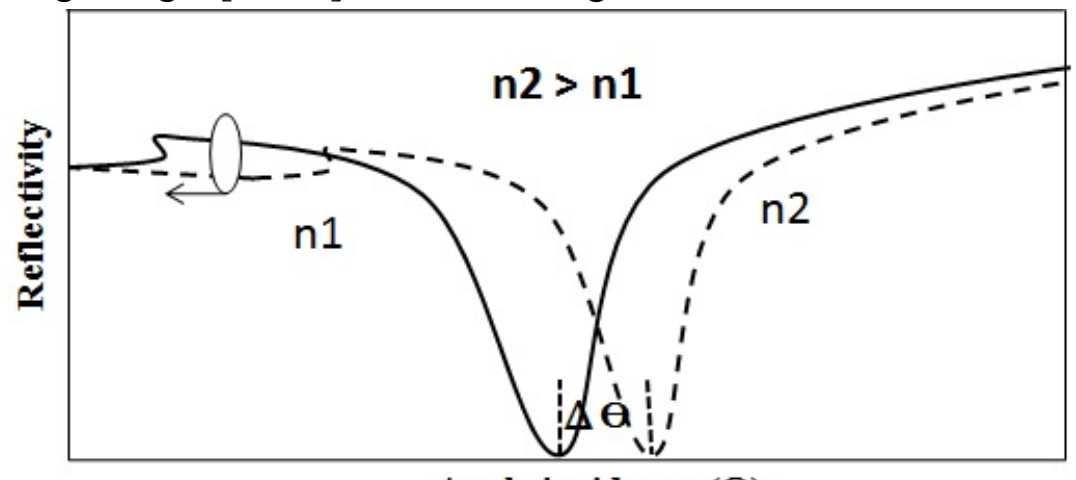

Angle incidence ( $\Theta$ )

Fig 2: Reflectance spectra as a function of incident angle $\left(\theta_{\text {inc }}\right)$ at the prism-metal interface

\section{SPR based bio-sensing}

Surface plasmon resonance has currently established as a potential optical tool for ultrasensitive detection of bioanalytes that could provide extraordinary low limit of detection. Compared to 
other types of electrical and optical sensors, it also showed added unique advantages of real-time and label-free sensing of biological samples in molecular level [33-37]. The development of SPR sensing devices for detection of various chemical and biological molecules has received significant impetus over the last couple of decades, for applications ranging from medical diagnostics [12], environmental monitoring [13] to food safety and defense security [14] etc. Many researchers are involved in exploring the possibility of the technology for fulfilling the recent demand of extremely high sensitivity of the order nano to pico molar concentration to very specific target which can open up new dimension of research in area such as drug discovery, food quality and safety, etc. [38-39]. The SPR biosensors are sensitive to refractiveindex changes at the metallic sensing substrate and known to be useful for the study of specificity, affinity, and kinetics of biomolecular interactions, and measurement of the concentration levels of target analytes in complex matrix samples. In the following section discussion are mainly focus onto Kretschmann's configuration in the study of SPR signals where the primary components are a prism based optical coupling system, SPR bio-chip and analyte handling system.

\subsection{SPR Bio-Chip}

Efficient immobilization and capture of bioreceptor on the sensing substrate is one of the critical steps in the development of optical biosensor. The sensor performance depends significantly on the appropriate surface treatment for effective immobilization of the bio-receptors on sensor surface. Up to date, most of the SPR detection schemes are adopted the self-assembled monolayers (SAMs) to conjugate small biomolecules such as peptides, DNA sequences and antibodies to the sensor surface. Index matched cover slips are typically used as the substrate for immobilization of the antibodies. After properly cleaning the cover slips, a thin layer of gold, silver or other metal supporting surface plasmon resonance is sputtered over it followed by biofunctionalization of the surface with antibody.

\subsubsection{Immobilization of antibody: functionalization, activation and binding of receptor}

Immobilization of properly-oriented antibody on the sensor surface is a measure of effectiveness of any immunosensors [40-43]. During immobilization, the antibody must retain their conformation. Moreover, the binding sites of the immobilized antibody should remain exposed to the antigen in order to ensure efficient antigen binding capacity. Direct immobilization on sensor surface results in random orientation of the immobilized antibodies, and thus make binding sites inaccessible for specific interaction with target antigen. This reduces their binding ability resulting poor sensitivity of the sensors [41,44]. Availability of highly dense active surface group of immobilized protein also influences the sensitivity of SPR sensor. Immobilization protocol therefore must ensure placement of properly oriented antibody on sensor surface with active binding sites exposed to the target sample. A great variety of immobilization strategies is 
reported that can be classifies as: physical, covalent and bio-affinity immobilization techniques. Physical adsorption presents the simplest method that utilizes ionic bonds or hydrophobic and polar interactions for immobilization. However, this binding appraoch yields poor reproducibility due to random orientation and denaturation of immobilized antibody [45, 46]. Contrary, covalent and bio-affinity based immobilization method provides higher stability and gently oriented antibody.

Conventionally used gold-coated SPR substrates generally lack sufficient active groups that are needed for direct attachment of antibody [40]. The inorganic metal surfaces are therefore activated with certain active groups that can efficiently bind with specific moieties $\left(-\mathrm{NH}_{2},-\mathrm{SH}\right.$, $\mathrm{COOH}$ etc.) of the protein to be immobilized. However, before such activation process, the inorganic surfaces are covered with protein-resistant self-assembled monolayer (SAM) in order to maximize the density of active surface groups [47-48]. In addition, SAM provides stable covalent binding, better control on orientation of immobilized proteins and helps in the minimization of non-specific protein adsorption on SPR chip [41, 49]. SAMs are ordered molecular assemblies that consist of active functional head group at either end of a hydrocarbon chain and a linear carbon chain that promotes self-assembly, when they are attached to a substrate surface. These active head groups have strong affinities for the binding surface while the other end remains free to bind with moieties of antibody.

Monolayer of alkanethiols on gold is the most extensively studied SAM for protein immobilization $[50,51]$. The thiol groups $(-\mathrm{SH})$ are known to exhibit strong binding affinity to the noble metallic surfaces that could allow them to form covalent bonds between the sulphur group in the thiol molecules and the metal atoms as shown in Figure 3. Alkanethiols with acid end group offers excellent chemical modification ability of the SAM surface making them promising for sensing applications [52-54]. SAM formed from 11-mercaptoundecanoic acid (11MUA) has been reported to form well-ordered monolayers by various research groups and is used for all the studies reported in this chapter [51,55].

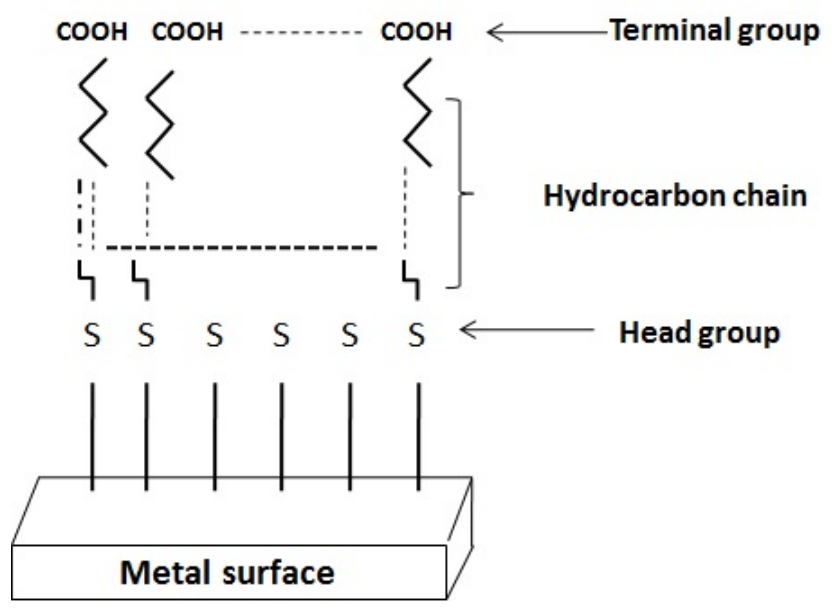


Figure 3: Representation of SAM of alkanethiols on metal

After surface functionalization for target antigen, the monolayer surface is required to be activated for facilitating the covalent attachment of the antibody using moieties such as amine, carboxylic, thiol, carbohydrate available in the protein. Among these functional groups, amine ($\mathrm{NH}_{2}$ ) groups in the lysine residue of the antibody have been widely used as anchoring points with their presence on the exterior of the antibody. Table I summarized the common functional groups in antibody used for covalent attachment and the substrate requirement. In the following section, amine based chemistry for antibody immobilization is presented which we have used in all further studied presented here.

Table I: Summary of commonly available functional groups for protein immobilization

\begin{tabular}{|l|l|l|l|}
\hline $\begin{array}{c}\text { Functional group in } \\
\text { protein }\end{array}$ & \multicolumn{1}{|c|}{ Amino acid } & Substrate requirement & \multicolumn{1}{|c|}{ Reference } \\
\hline Amine $\left(\mathrm{NH}_{2}\right)$ & Lysine & $\begin{array}{l}\text { Carboxylic acid active } \\
\text { ester }\end{array}$ & $56-58$ \\
\hline Carboxylic (-COOH) & $\begin{array}{l}\text { Aspartate, } \\
\text { Glutamate }\end{array}$ & Amine & 59 \\
\hline Thiol (-SH) & Cysteine & $\begin{array}{l}\text { Maleimide, pyridyil } \\
\text { disulfide }\end{array}$ & $60-62$ \\
\hline Carbohydrate $(-\mathrm{COH})$ & $\begin{array}{l}\text { Sugar residue after } \\
\text { oxidation }\end{array}$ & Silane modified surface & 63 \\
\hline
\end{tabular}

Amine based coupling chemistry with carboxylated gold surface is shown in Figure 4. This popularly used carbodiimide chemistry has been applied to produce robust amide bonds between the amine groups and the carboxyl groups of the antibody. Water-soluble carbodiimide reagents 1-ethyl-3- (3-dimethylaminopropyl)-carbodiimides (EDCs) are used to activate the carboxyl group present on the substrate in order to produce a reactive $O$-acylisourea intermediate. When N-hydroxy succinimide (NHS) is added, the nucleophile of NHS reacts with the intermediate $O$ acylisourea and forms an NHS ester that is much more stable than the $O$-acylisourea intermediate and leads to their efficient conjugations to primary amines [64-65]. The conjugation between aldehyde and amine groups realized the immobilization of antibody by a labile Schiff's base formation. 


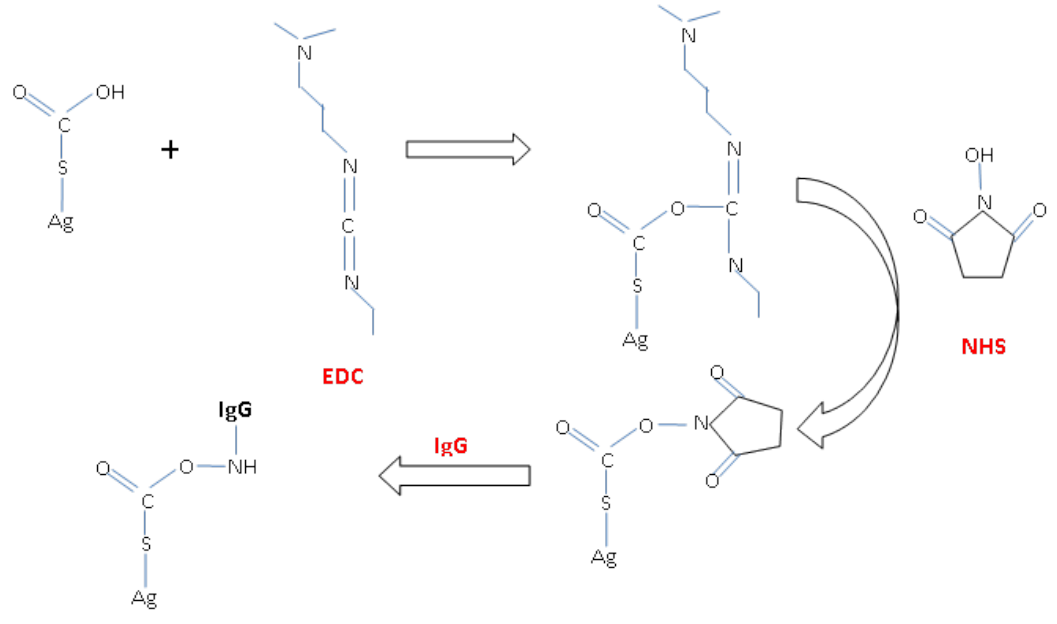

Figure 4: Amine based coupling chemistry

\section{Immobilization Protocol:}

The following protocol is a standard process used for the preparation of SPR sensor with immobilized antibody [56, 66-67].

\section{A. Substrate preparation:}

1. The microscopic cover slips $\left(18 \times 18 \mathrm{~mm}^{2}\right)$ were cleaned using acetone vapor at $80{ }^{\circ} \mathrm{C}$ followed by treatment with methanol at room temperature for five minutes each. Finally, the slides were rinsed in Deionized (DI) water and then dried in a nitrogen environment.

2. Place the samples onto a hotplate with faceing up at $180^{\circ} \mathrm{C}$ for about 15 mins.

3. Sputter deposit $\sim 50 \mathrm{~nm}$ gold or silver layer. A thin layer of titanium or chromium $(\sim 5 \mathrm{~nm})$ is required to be deposited first before the deposition of $\mathrm{Au} / \mathrm{Ag}$ layer in order to improve the adhesion capability of $\mathrm{Au} / \mathrm{Ag}$ with glass.

\section{B. Functionalization followed by activation of sensor surface:}

1. Prepare separate solutions of $1 \mathrm{mM}$ ethanoic solution of 11-MUA, $75 \mathrm{mM}$ EDC, $15 \mathrm{mM}$ NHS and $50 \mathrm{mM}$ ethanolamine solution in water.

2. Dip the metal-coated substrates in the ethanoic solution 11-MUA in a close container for 24 hours at room temperature.

3. Rinse the MUA coated chip in ethanol and dry under nitrogen stream.

4. Drop $100 \mu \mathrm{L}$ of EDC and NHS successively using a micropipette over the sample.

5. Incubate the sample for one minute in a tightly closed petri dish.

\section{Antibody immobilization}

1. The slide was immersed into anti-mouse $\operatorname{IgG} 2 \mathrm{~b}$ solutions/ $\operatorname{IgG}$ in $50 \mu \mathrm{L}$ phosphate buffer solution (PBS) at $\mathrm{pH}$ of 7.4 for 2-4 hour to form stable monomolecular layer 
2. The free amine groups of EDC-NHS from the surface functionalization process are blocked through immersing the SPR chip in solutions containing $50 \mathrm{mM}$ ethanolamine $(100 \mu \mathrm{L})$ for 10 to 20 mins to avoid the unwanted binding of free amine group from EDC-NHS with target molecules.

3. Finally, the chip is washed gently with $0.5 \mathrm{~mL}$ PBS and then kept at $4{ }^{\circ} \mathrm{C}$ for 12 hours.

\subsubsection{Characterization of sensor surface}

The immobilization protocol for SPR sensor involves a number of steps and the sensor surface consists of a number of layers staked together. The surface composition and structure of the deposited layers viz., thickness of the metal layer, morphology of SAM layer for surface functionalization and NHS-EDC for surface activation is essential for ensuring properly oriented immobilization of antibody. The immobilization of the antibody is usually characterized by spectroscopic measurements such as Fourier-transforms infrared spectroscopy (FTIR) and ultraviolet-visible spectroscopy (UV-Vis) in our studies as other research groups did. However, various other methods can also be used for assessing the state of immobilized antibody, doing elemental and chemical characterization and studying the surface morphology of the stacked layers. Zhao et al., reported a detailed study on surface composition and structure of selfassembled thiol-monolayer of alkanethiol molecules using X-ray photoelectron spectroscopy (XPS), FTIR and energy dispersive spectroscopy (EDS), [68]. A summary of various characterization techniques is presented by Welch et al.[42].

Figure 5 shows the optical absorption spectra using by a UV-Visible spectroscopy of the SPR sensing substrate before/after antibody (IgG) immobilized onto pure silver (Ag) thin film deposited sensor surface. As one can see, the absorbance peak of pure Ag film is centered at 310 $\mathrm{nm}$, and the following deposition of the additional molecular layer on the silver nanofilm results in a red shift in the SPR dip. The surface functionalization with an 11-MUA layer led to the absorption peak with a redshift by $\sim 5 \mathrm{~nm}$ that is attributed to the covalent binding of thiol molecules to the metallic surface. While when the antibody is further conjugated, a redshift of 15 $\mathrm{nm}$ was induced on the absorbance peak. This is due to the increase of the local dielectric constant of at the metal interface by an additional molecular layer. 


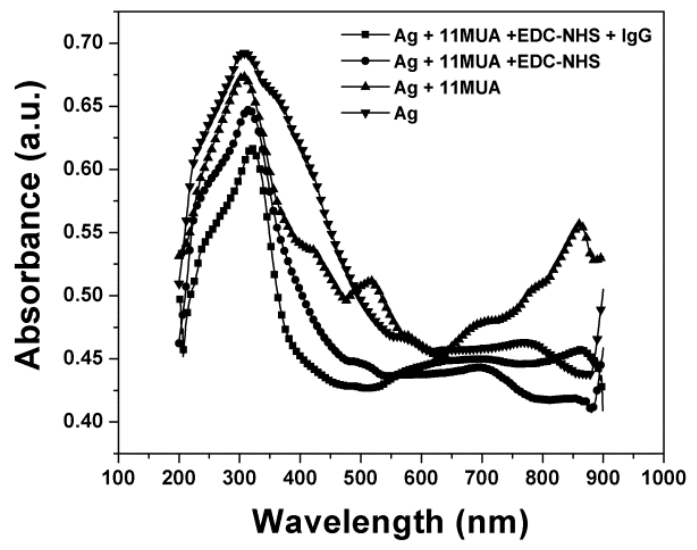

Figure 5: UV-Vis spectra of the pure silver (Ag) thin film deposited sensor surface before and after antibody (IgG) immobilized; reprinted with permission form Choudhury et al., Creative Commons Attribution 4.0 International License (http://creativecommons.org/licenses/by/4.0/).

The FTIR measurements are performed with Nicolet Impact 410 to characterize the chemical structures of the SPR surface. Figure 6 presents the infrared spectroscopy (IR) spectra of the sensing substrate after the monolayer binding with 11-MUA, followed by surface activation with NHS-EDC, and lastly target antibody conjugation. The end chemical groups of 11-MUAs are carboxyl groups of - $\mathrm{COOH}$. The amine groups of NHS conjugated to this group through reaction with EDC finally formed bond of COO-NHS. After the capture process of target antibodies, the end group is then converted to COO-N-IgG as well. Figure 6a also showed the band region for carbon inside the IR spectra. This carbon region was characterized through two absorption peaks at wavenumbers $2932 \mathrm{~cm}^{-1}$ and $2856 \mathrm{~cm}^{-1}$ respectively that generated by the $\mathrm{CH}_{2}$ symmetric and asymmetric stretches [69]. Figure $6 \mathrm{~b}$ presents the amide and carboxyl region inside the IR spectra. In comparison to the 11-MUA molecules-functionalized films, NHS terminated-film showed three characteristic bands centered at $1820 \mathrm{~cm}^{-1}, 1798 \mathrm{~cm}^{-1}$, and $1765 \mathrm{~cm}^{-1}$ that are responsible for stretch modes of COO-NHS esters. After the immobilization of the target antibody $\mathrm{IgG}$, there are two new amide bands appearing at $1570 \mathrm{~cm}^{-1}$ and $1687 \mathrm{~cm}^{-1}$ (amide I and amide II), which further confirmed the successful binding of IgG molecules on the substrate [70]. 

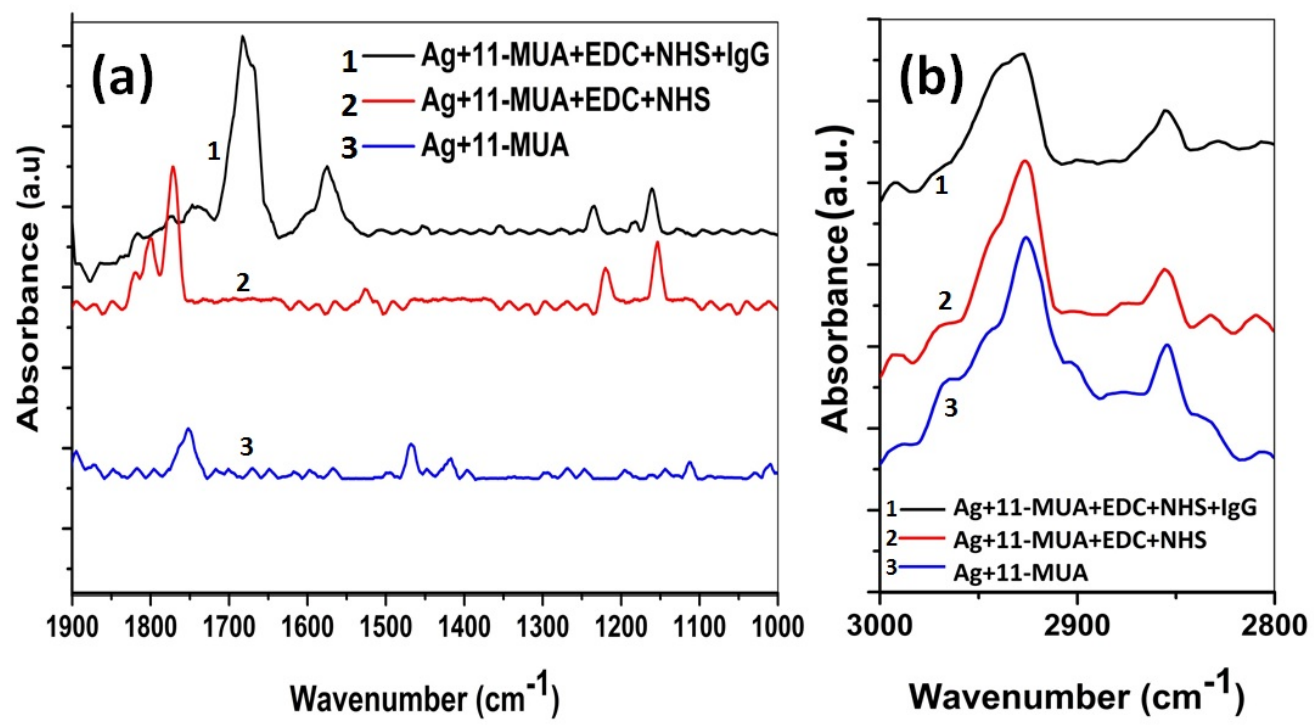

Figure 6: Fourier-transforms infrared spectroscopy (FTIR) spectra of the SPR sensing surface before/after the immobilization of target antibody IgG; reprinted with permission form

Choudhury et al., Creative Commons Attribution 4.0 International License (http://creativecommons.org/licenses/by/4.0/).

\subsection{SPR set-up}

\subsubsection{The optical assembly}

The conventional method of excitation of Surface Plasmon Resonance (SPR) based on Kretschmann's configuration involves focusing laser beam through a prism coated with metal layer which in turn is placed on a rotating stage. Surface Plasmon is excited at the interface between a metal and dielectric layer at particular incident angle of the light beam. A complex rotation mechanism is needed to adjust the incident angle of the incoming beam and to receive the reflected light. Prof. Mukherji and his group at Indian Institute of Bombay (IITB) have given significant contribution for the development of portable SPR measurement device [71]. At Tezpur University a number of experimental set-ups were also fabricated by our group and Figure 7 shows a raw version of one [72]. The device comprised of a BK7 prism which was mounted with two supports from its two sides in such a way that it could be easily removed/replaced whenever required. The laser diode $(650 \mathrm{~nm}, 5 \mathrm{~mW})$ was mounted on an "L" shaped leg and its movement was controlled using a servomotor to adjust the incident angle of the incoming light within an angular span of $45-80^{\circ}$ as a reference to the base of the prism. The incoming light from the laser diode went through a linear polarizer to generate a p-polarized light beam and made to fall on the sensor surface at varied angles of incidence. Glycerine having a refractive index unit (RIU) of 1.42 was used as the index-matching layer for sticking the microscopic cover slips on the glass prism (BK7) in order to avoid the any air gaps in between. A detector array from photodiodes is used to collect the light signals reflected from the prism surface. Alternatively, the reflected light from the sensor surface might be converged using a 
convex lens and collected with a charge-coupled device (CCD) camera based on a universal serial bus (USB) interface.

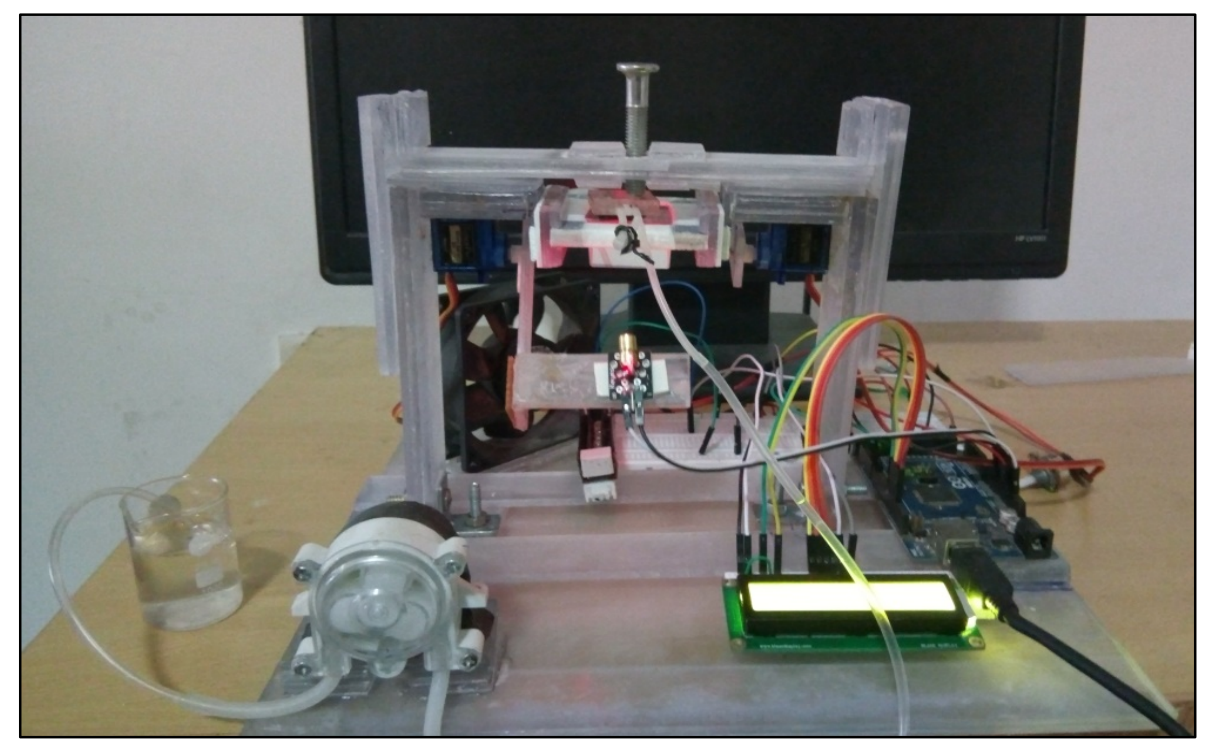

Figure 7: Snapshot of SPR measurement set up

\subsubsection{Analytes handling and sensor measurement}

The fluid handling system of the device consisted of a specially designed flow cell $\left(\sim 280 \mathrm{~mm}^{3}\right)$ regulated by peristaltic pump which was used to pass stream of test samples through the sensor surface. The flow cell could be readily attached to the sensor surface using O-ring to prevent leakage of test fluid. A schematic arrangement of fluid handling system is shown in figure. The operation of the pumping system was controlled using an Arduino microcontroller. Before the protein conjugation studies, the phosphate buffer solution (PBS) with a $\mathrm{pH}$ of 7.4 is flowed onto the self-assembled monolayer (SAM) for establishing a baseline signals for the following tests. For the subsequent measurement steps, the sensor head is reacted with target IgGs in different concentrations of $(0.05 \mathrm{mg} / \mathrm{ml}$ to $0.35 \mathrm{mg} / \mathrm{ml})$ diluted in the PBS solution for 10 mins. The antigen-antibody binding is depicted in Figure 8. For the final step, PBS is then injected to the sensing substrate to initiate the dissociation binding of protein molecules. 


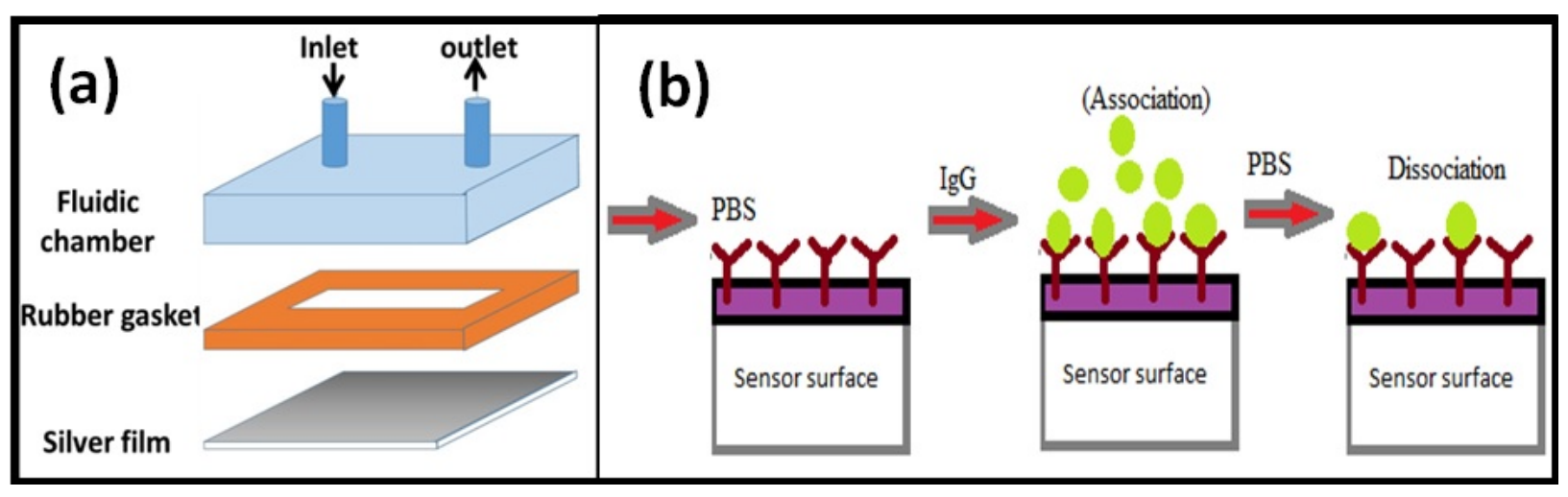

Figure 8: Schematic of fluid handling system (a) and sensor measurement procedure (b)

In modern biotechnology particularly for drug discovery, clinical and proteomic research, multiple proteins detection is becoming increasingly important where sophisticated fluidic systems for precise delivery of various fluids to the sensor surface is essential. In this direction Piliarik et al. have reported a SPR sensor with high sensitivity that consisted of a SPR Disperser (SPRCD) and a coupler. The sensor head is integrated with a set of microfluidic channels enabling the device for multiple measurements [73]. Using this compact system designed at the Institute of Photonics and Electronics, Prague (Czech Republic), portable SPR immunosensors with six channels for simultaneous detection of antibiotics in milk sample was reported by Fernandez et al [74]. Recently Liu et al. also demonstrated multi-analyte detection capability of a compact multichannel SPR biosensor even on a cell mobile device as an effective platform for in-situ measurement [75].

\subsection{Experimental result}

The value of intensities of the light reflected from the SPR sensing chip as a function of the incident angles are plotted in Figure 9 below. Protein $\mathrm{IgG}$ in a concentration of $0.05 \mathrm{mg} / \mathrm{ml}$ is flowed and reacted with the capture antibody (monoclonal anti-IgG) immobilized sensor surface. The redshift of the SPR resonance dip with successive additional deposition of layers over the sensor surfaces indicates binding between the stacked layers. 


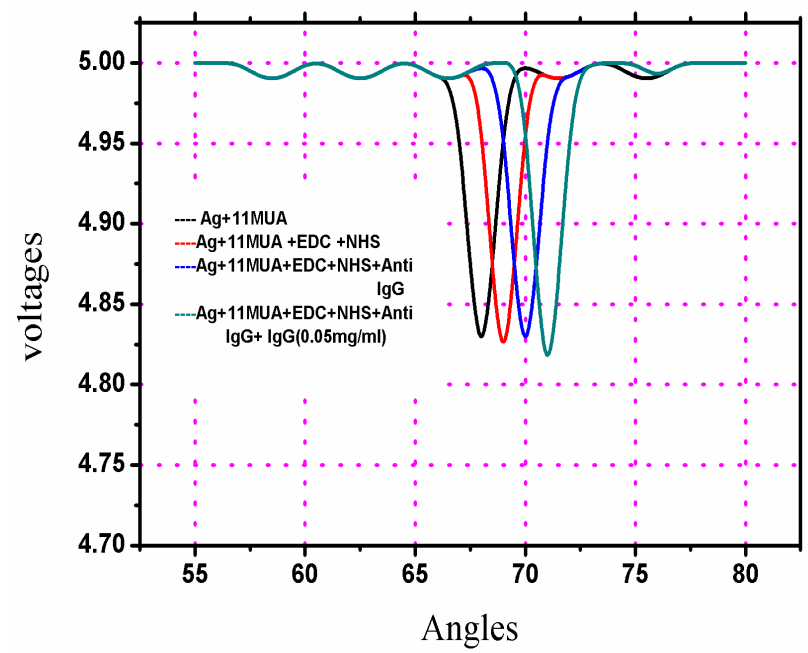

Figure 9: SPR measurements on the stacking sandwich layers.

To systematically study the antigen-antibody interactions, antibody proteins with different concentrations were flowed and reacted with the IgG-functionalized sensing substrate. All of the measurements are carried out by immersing the sensor head with the protein diluted in the PBS buffer. The output data with dip angle in terms of the IgG concentrations are plotted in Fig 10 (a, b). The flow of protein of higher concentration results in higher degree of refractive index change in the medium close to the sensor interface. This is due to more binding events between antigen-antibody. The binding process made the resonance dip to shift towards right with increasing concentration of the target protein as can be from fig 10(a). It is worth noting that the SPR characteristic curve is not much affected by change in the concentration of the sample, as the full width half maxima (FWHM) remain nearly identical for all the different samples.
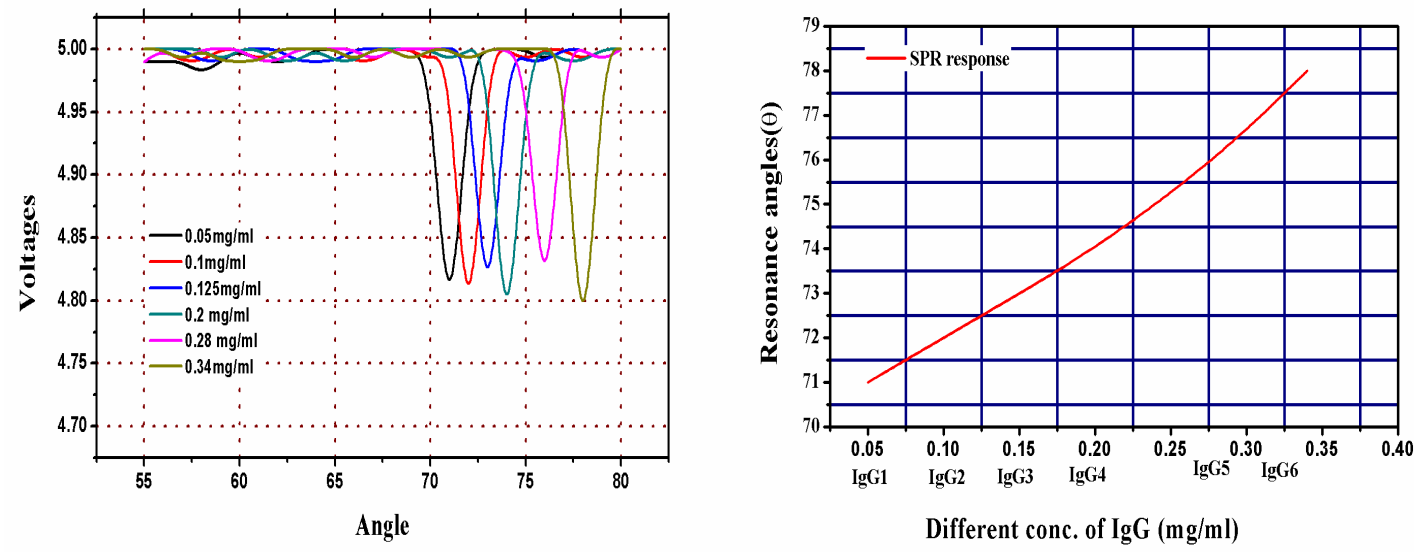

Figure 10: Experimentally measured SPR data with varied protein concentration.

The SPR sensorgrams depicting association and dissociation for the sensor is shown in Figure 11. The baseline was initially measured by flowing PBS through the monoclonal anti-IgG 
functionalized sensor surfaces for a period of 5 minutes. While the continuous flow of protein, the sensor signal reaches a maximum and the sensor is defined in the association phase during this period. A relatively low concentration $(0.05 \mathrm{mg} / \mathrm{ml})$ target protein is initially exposed to the sensor resulting in the shift of the resonance tip towards right. After twelve minutes when the injection of protein is stopped, the sensor slowly goes to a steady state (saturate) level. Later, the sensing signal returns to a value close to the one of baseline the during the dissociation phase when a stream of PBS is passed through the sensor surface. Subsequently, the cycle is repeated by increasing the concentration of the target protein that shows good repeatable behavior of the sensor.

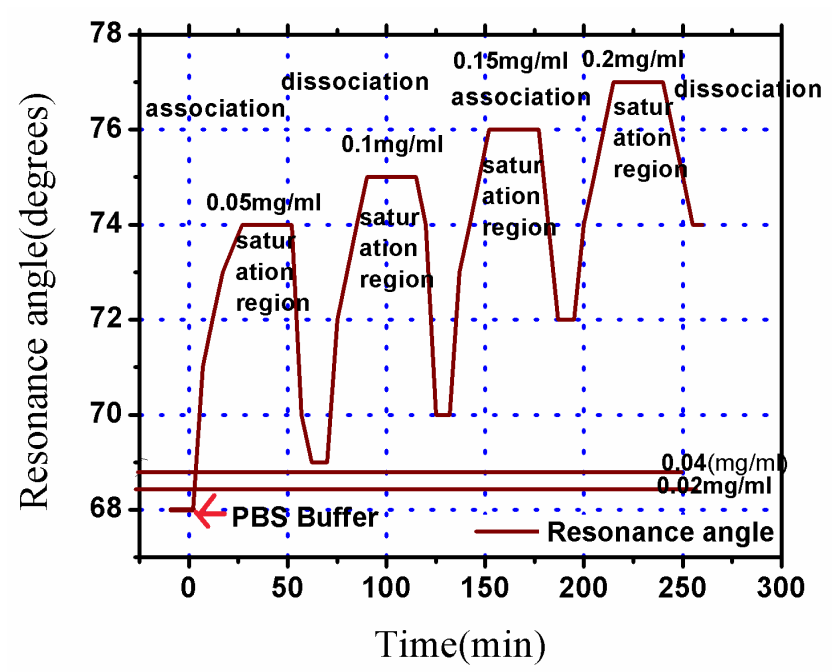

Figure 11: SPR sensorgrams showing binding kinetic with protein IgG of different concentrations.

\section{Performance characteristics of SPR-based sensing}

The performance parameters of a SPR sensing device are generally investigated by: its sensitivity, accuracy of measurement and limit of detection. The SPR sensitivity with angular interrogation is defined as: $S=\delta \theta_{\text {res }} / \delta n_{s}$, where $\delta \theta_{\text {res }}$ is the resonance angle shift due to the refractive index change of the sensing medium by $\delta n_{s}$. Sensitivity enhancements with various methods such as the integration of plasmonic nanostructures coupled onto SPR chip [76], use of nanogratings [77] and graphene [78] etc., have been reported. The characteristics of the SPR curve including its shape, full width at half maxima (FWHM), position of the SPR resonance dip etc., are of significant importance for sensing applications. A smaller value of FWHM implies a high signal-to-noise $(\mathrm{S} / \mathrm{N})$ ratio signifying better accuracy/resolution of measurements [79]. Figure-of-merit (FOM) is known as the ratio between sensitivity (S) and the FWHM. Various research groups also use it as a key parameter to evaluate sensing performance of SPR sensor. 
Uses of absentee layer, or high refractive-index thin dielectric layer, bimetallic layers were found suitable for FOM enhancement [80-82]. Limit of detection (LOD) is another key parameter and is usually termed as the minimum input quantity measured with higher than $99 \%$ fidelity. The LOD could be calculated by $3 \in / \mathrm{S}$, in which $\in$ is the uncertainty of output [83].

\subsection{Choice of metal for SPR sensor}

The choice of the metal layer and its film thickness has significant impact on the performance characteristics of the SPR sensor. Silver and Gold are usually the preferred choices for the metal layer due to their unique properties such as: the high efficiency in the electron resonance at the conduction band for specific excitation wavelength, thermal/chemical stability, and ability to conjugate organic molecule for surface functionalization. It has been observed that silver film yields a more distinct SPR spectrum than gold. A thin film of silver with thickness around $50 \mathrm{~nm}$ produces a sharp SPR signal supporting precise determination of the resonance angle [84]. Gold on the other hand produces a larger shift of the resonance angle than that of silver for a fixed change of refractive index unit of the sensing layer, while it is much more stable in the ambient environment and more resistant to the oxidization [85]. However, a bimetallic film of silver-gold can combine the advantages of both materials thereby accounting for better SPR sensing. Zynio et al were the first research group to propose bimetallic films of gold and silver. In their designed structures, gold was deposited on top of silver to for the excitation of SPR. It was reported that the bimetallic films displayed a large shift of resonance angle due to the changes of refractive index similar to gold thin films as well as showed a narrow resonance curve like silver, thereby leading to a high detection accuracy of measurements. In addition to it, the silver was protected against oxidation by the outer gold layer [86]. Yuan et al also presented a bimetallic configuration of silver-gold. In their results, the obtained value of Full Width at Half Maximum (FWHM) with the bimetallic film was found to be much smaller than that of the pure gold film, while the stability of the bimetallic film was found to be better than that of the pure silver film [87]. In our recent study, we also emphasized on parameters such as full width at half maxima (FWHM) and sensitivity of a set of three bimetallic sensors are prepared by varying the metal film type and thickness [88]. The samples and their specifications are indicated the Table II.

Table II: Sample ID with Specifications

\begin{tabular}{|c|c|c|}
\hline Sample ID & $\begin{array}{l}\text { Name of the } \\
\text { metallic film }\end{array}$ & Thickness of film \\
\hline I & Silver & $50 \mathrm{~nm}$ \\
\hline II & Gold & $50 \mathrm{~nm}$ \\
\hline
\end{tabular}




\begin{tabular}{|l|l|l|}
\hline III & Silver-Gold & $\begin{array}{l}35 \mathrm{~nm} \text { (Silver) and } \\
15 \mathrm{~nm} \text { (Gold) }\end{array}$ \\
\hline
\end{tabular}

Figure 12 shows the SPR responses of the silver, bimetallic and gold based sensor chips towards three test samples (Water, Acetone and Butanol). The experimental results of FWHM obtained from the graphs in figures are listed in Table II. From the FWHM values it was seen that pure silver had the least FWHM due to its narrowest width of the curve, thus providing the highest resolution of detection of the resonance angle. In comparison, the width of the pure goldbased sensor chip is the largest among these three configurations. As far as the bimetallic sensor chip was investigated, it exhibited a small value of FWHM (approximately 6 times less than the pure gold based sensor chip) indicating reasonably better detection resolution of the resonance angle. As mentioned above, the SPR Sensitivity parameter is dependent on the value of the resonance dip (angular shifts) for a fixed refractive index change of the surrounding sensing media. If the angular shift is a larger signal change, the SPR sensitivity is considered to be relatively higher. Table III lists the experimental measurement results showing the signal change of the resonance dip angle (in degrees) due to the corresponding refractive-index change of the sensing layer extracted from the graphs in Figure 12.
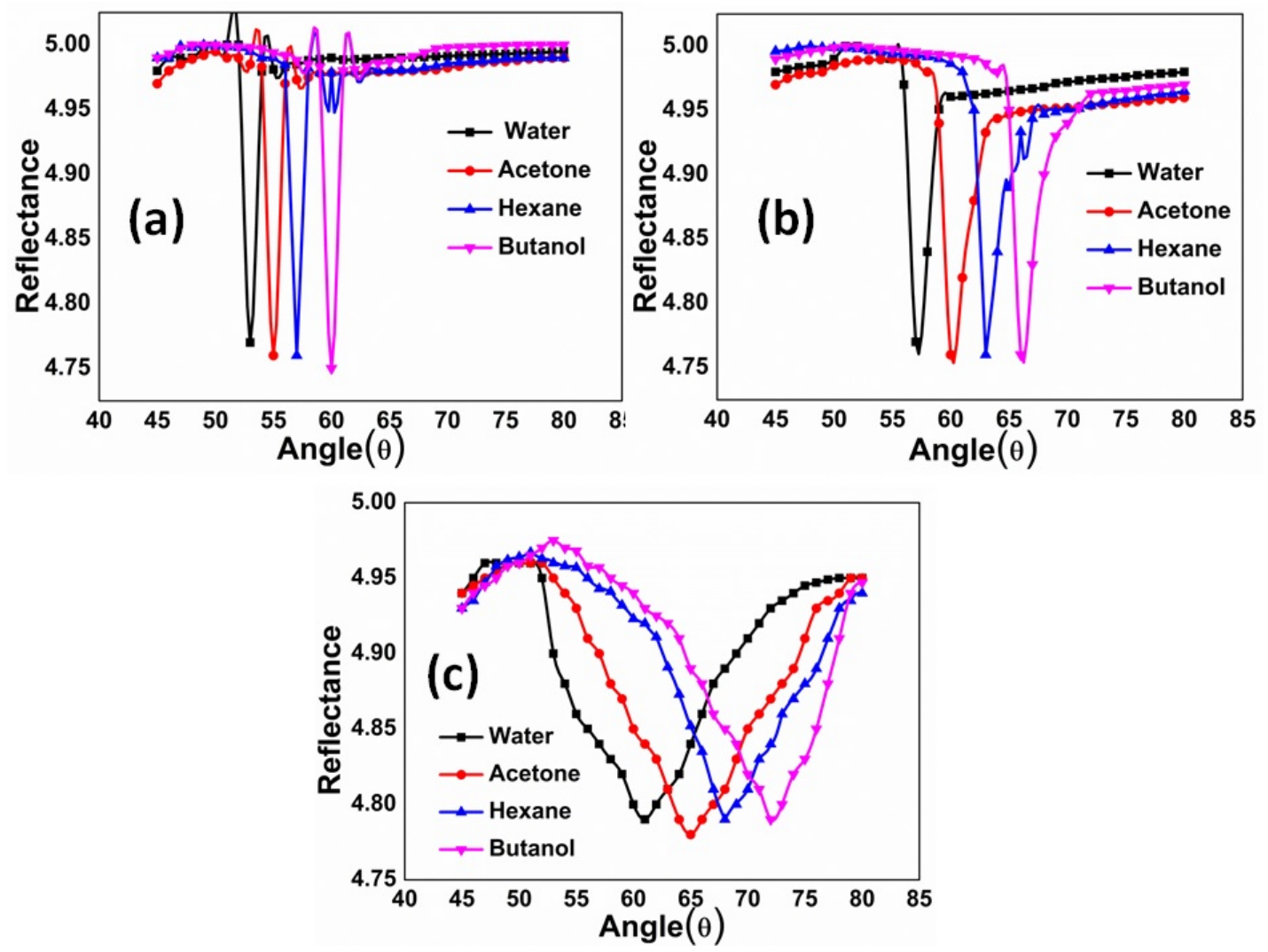

Figure 12: SPR responses of the silver (a), bimetallic (b) and gold (c) sensor chip towards Water, Acetone, hexane and Butanol. 
Table III: Experimental results of FWHM (in degrees) for different configurations

\begin{tabular}{|l|l|l|l|}
\hline $\begin{array}{l}\text { Test } \\
\text { Samples }\end{array}$ & Silver & Silver-Gold & Gold \\
\hline Water & $1^{\circ}$ & $2^{\circ}$ & $12^{\circ}$ \\
\hline Acetone & $1^{\circ}$ & $2.5^{\circ}$ & $12^{\circ}$ \\
\hline Hexane & $1^{\circ}$ & $2^{\circ}$ & $11^{\circ}$ \\
\hline Butanol & $1^{\circ}$ & $2^{\circ}$ & $11^{\circ}$ \\
\hline
\end{tabular}

Table IV: Shift of the resonance angle due to change in refractive index of the sensing layer

\begin{tabular}{|c|c|c|c|}
\hline \multirow{2}{*}{$\begin{array}{l}\text { Refractive } \\
\text { index unit } \\
\text { (RIU) }\end{array}$} & \multicolumn{3}{|c|}{$\begin{array}{l}\text { Shift in Resonance Angle (in degree) } \\
\text { For sensor with }\end{array}$} \\
\hline & Silver & Silver-Gold & Gold \\
\hline 0.0261 & $2^{\circ}$ & $3.5^{\circ}$ & $4^{\circ}$ \\
\hline
\end{tabular}

\subsection{Sensitivity enhancement with graphene, $\mathrm{MoS}_{2}$ and 2D Perovskite nanolayers}

As introduced in the previous sections, plasmonic sensors are known as an efficient tool for real time monitoring biomolecular interactions. The detection mechanism is based on the field perturbation at the plasmonic sensing interface induced by the binding of molecules. This molecular binding process could be recorded through reflected light signal when the surface plasmon is excited by the incident light. In recent studies, we have presented the use of hybrid 2D nanomaterial-based metasurface nanostructures as a plasmonic sensing substrate. The thickness of the sensing substrate is tuned in an atomic scale and optimized to obtain an enhanced sensing effect. More specifically, a sharp phase signal change and phase-related GoosHänchen signal shift was achieved that results from the strong resonance. The improved sensitivities of $2 \mathrm{D}$ nanostructures were investigated. It is worth noting that the atomic layer design led to the sensing substrate optimized with a tuning scale less than $1 \mathrm{~nm}$. By precisely design the metasurface substrates, more than 4 orders of magnitude improvement of the sensitivity $(900,000 \mathrm{um} / \mathrm{RIU})$ were obtained in comparison to the one with pure gold sensing substrate (400 um/RIU). 


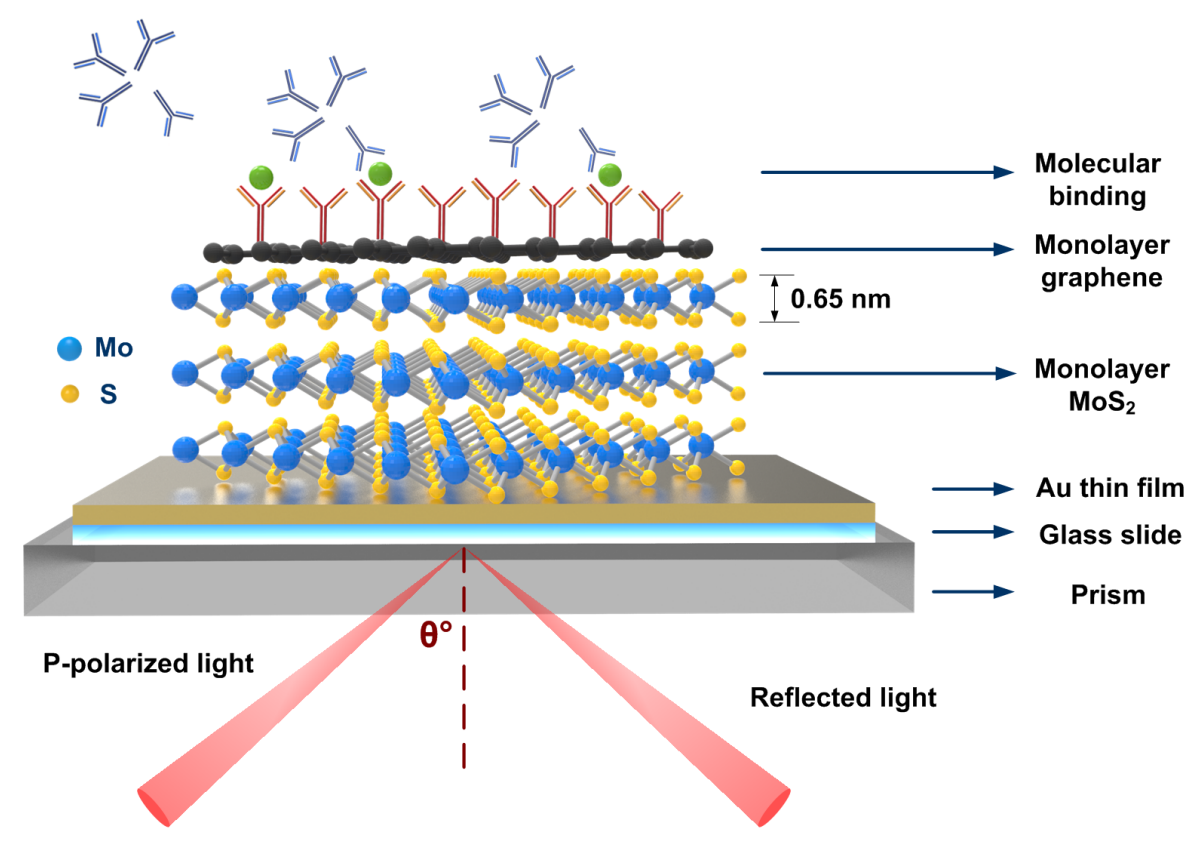

Figure 13: Schematic configuration of the designed graphene/MoS 2 nanolayers on $45 \mathrm{~nm}$ gold sensing substrate for protein detection [89].

Figure 13 illustrates the configuration of our designed $2 \mathrm{D}$ graphene/ $\mathrm{MoS}_{2}$-enhanced plasmonic biosensors. A single layer of graphene is deposited on the top of the sensing substrate to capture the target biomolecules. The hexagonal carbon ring structure of graphene could enhance the adsorption rate of the analyte due to pi-stacking force. It is worth noting that monolayer hexagonal boron nitride ( $\mathrm{hBN}$, known as white graphene) could also be coated on the silver thin film to protect the surface from oxidization during the detection process. It is known that under the similar resonance condition (e.g., excitation wavelength, thickness of metallic thin film, SF11 prism coupling), the silver thin film exhibited a better sensing performance with high sensitivity and high detection resolution than that of gold ones, in terms of a lower minimum reflectivity and narrower width of the reflectance spectra. Graphene is measure to be the thinnest twodimensional nanomaterial so far in the world. Since its first report in 2004 by Andre Geim and Kostya Novoselov, their unique optical and electronic properties have been demonstrated such as its zero bandgap with high electrical conductivity, and zero effective mass at the Dirac point. The high charge carrier mobility of graphene up to $10^{6} \mathrm{~cm}^{2} \mathrm{~V}^{-1} \mathrm{~s}^{-1}$ makes it an attractive candidate for the next generation of substrate for SPR sensors. By designed the graphene layers on the gold or silver SPR sensor head, strong electric field enhancement at the hybrid interface would be generated through an effective charge transfer process. The drastic concentration of plasmon electric field in this 2D plane provides a novel sensing functionality and makes it fit to the definition of "metasurfaces". In our recent studies, we have demonstrated an ultrasensitive graphene-based surface plasmon resonance sensing device with a detection limit as low as attomolar $1 \mathrm{aM}\left(10^{-18} \mathrm{M}\right)$ for target single-stranded DNA (ssDNA) analytes as shown in Figure 14 was demonstrated by our group [90]. 
a

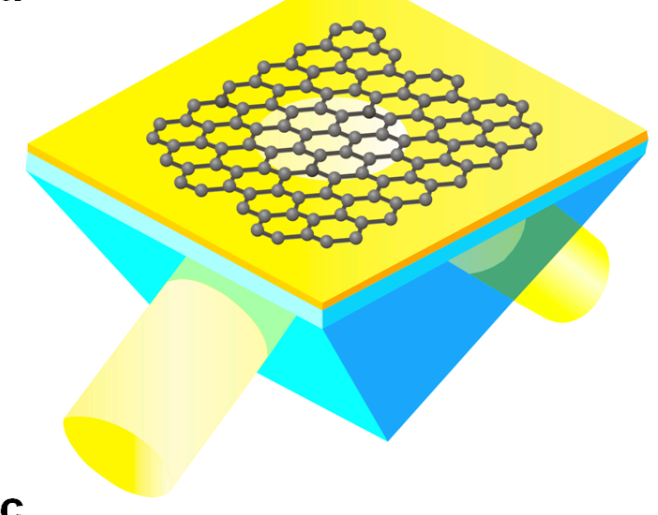

C

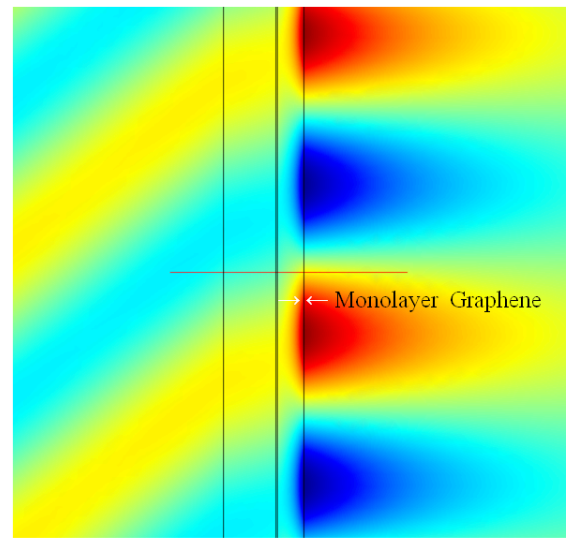

b

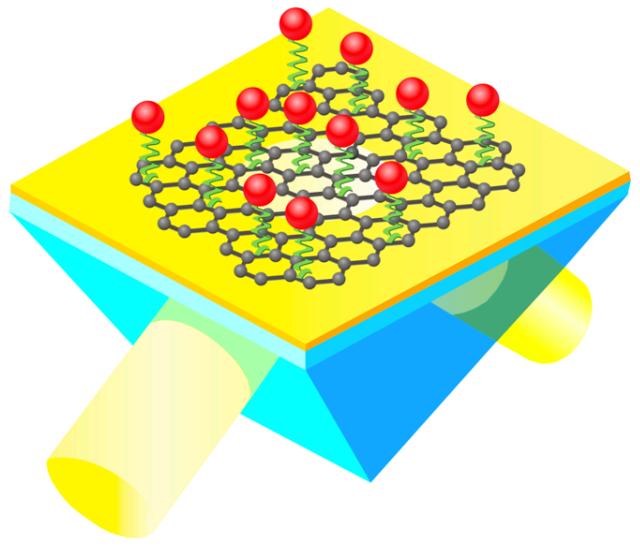

d

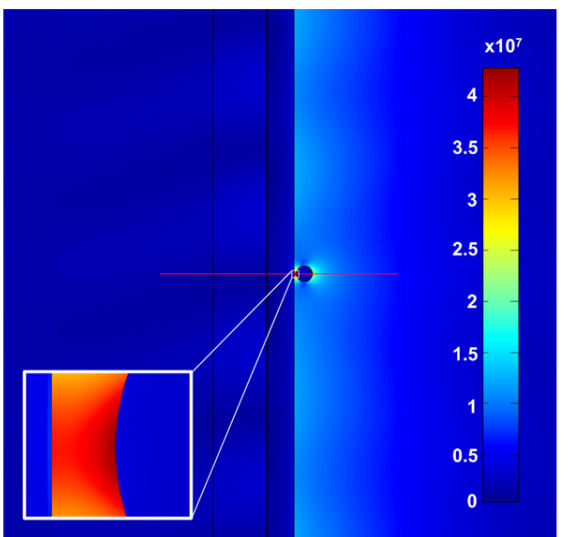

e

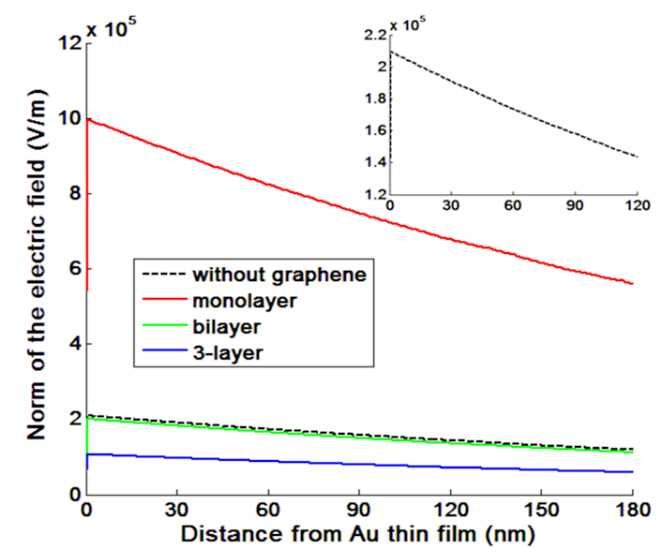

Figure 14: Designs and sensing results of graphene-gold metasurface architectures [90]. 


\section{Summary and Outlook}

Two-dimensional (2D) transition metal dichalcogenides (TMDCs) nanolayers have become intriguing to the SPR community and showed their potential to improve the performances of different optoelectronic devices such as solar cells and photo detection arrays [91-94]. It is worth noting that absorption rate $(\sim 5 \%)$ is the much higher for TMDC nanomaterials than that of monolayer graphene $(2.3 \%)$. The thickness is also with the atomic thickness range. 2D TMDCs showed a tunable bangdap characteristic that is different from that of graphene. Their bandgaps could be tuned from indirect to the direct ones when their thicknesses are down to the nanoscale. This feature promises them to be complementary with graphene for the design of next-generation integrated flexible optoelectronic devices. The physical structure of TMDCs nanomaterials are similar to the graphene in which the metal atom $\mathrm{M}$ and the chalcogen atoms $\mathrm{X}$ were bonded in a hexagonal plane. As of the TMDC in the bulk forms, the stacking of each layer was achieved by van der Waals forces. Thus, monolayer TMDC can be obtained by exfoliation. Up to date, the atomic thin TMDC with large size have been fabricated through vapor deposition growth. To achieve multiple layer substrates, one could transfer the single layer and repeatedly stack them on the target sensing substrate. Several research groups have reported that depositing a thin layer of dielectric materials could significantly vary the SPR resonance condition and thus may facilite on the integration process of the current prism-based plasmonic sensors [95-96]. The fabrication of atomically thin 2D nanomaterials has been reported in recent literatures [91-94]. There are different types of TMDC materials including $\mathrm{MoS}_{2}, \mathrm{MoSe}_{2}, \mathrm{WS}_{2}$ and $\mathrm{WSe}_{2}$, which depend on the combination of its metal and chalcogen elements. The presence of 2D TMDC metasurface layers could enhance the transmission loss, which provides stronger surface plasmon resonance.

In addition to the novel 2D nanomaterials as enhanced sensing susbtrate, the SPR detection schemes could also be step into the next generation instead of the traditional angular and wavelength scanning method. Since Surface plasmon resonance (SPR) only affects $p$-polarized light, which is parallel to the propagation direction. Therefore $s$-polarized light can be used as a reference signal. Based on this mechanism, we could extract the differential signal between the two polarized light beams from the reflected side simultaneously. The environmental noise and the stability of the setup can be significantly improved during experimental measurement. The signals that we collect are phase-related Goos-Hänchen signals reflected from the sensing substrate, which is a lateral position shift of the light beams at the sensing interface. The GH detection is different from the angular measurement of the conventional method, which suffers from the loss of the metallic and graphene materials. The phase and GH jumps are only corresponding to the dip value of the SPR reflectance curves. With gold nanoarray in Figure 15, the field coupling of localized SPR and SPP waves would further improve the detection sensitivity. As the concentration of sensing medium increases, groove metasurface-based SPR sensor undergoes larger GH signal shift. Integration of fiber-device based on nanostructuremetasurface (e.g. transverse Anderson localization and Fano resonance) for enhanced plasmonic 
sensing and image transport is another future trend for the development of portable SPR biomedical devices. One of the highlights is our study [98] on the integration of functional gold nanoparticles with optical micro/nanofibers (OMNFs) as a versatile platform for sensing of streptavidin biomolecules with a low detection of $1 \mathrm{pg} / \mathrm{ml}$ in Figure 16.
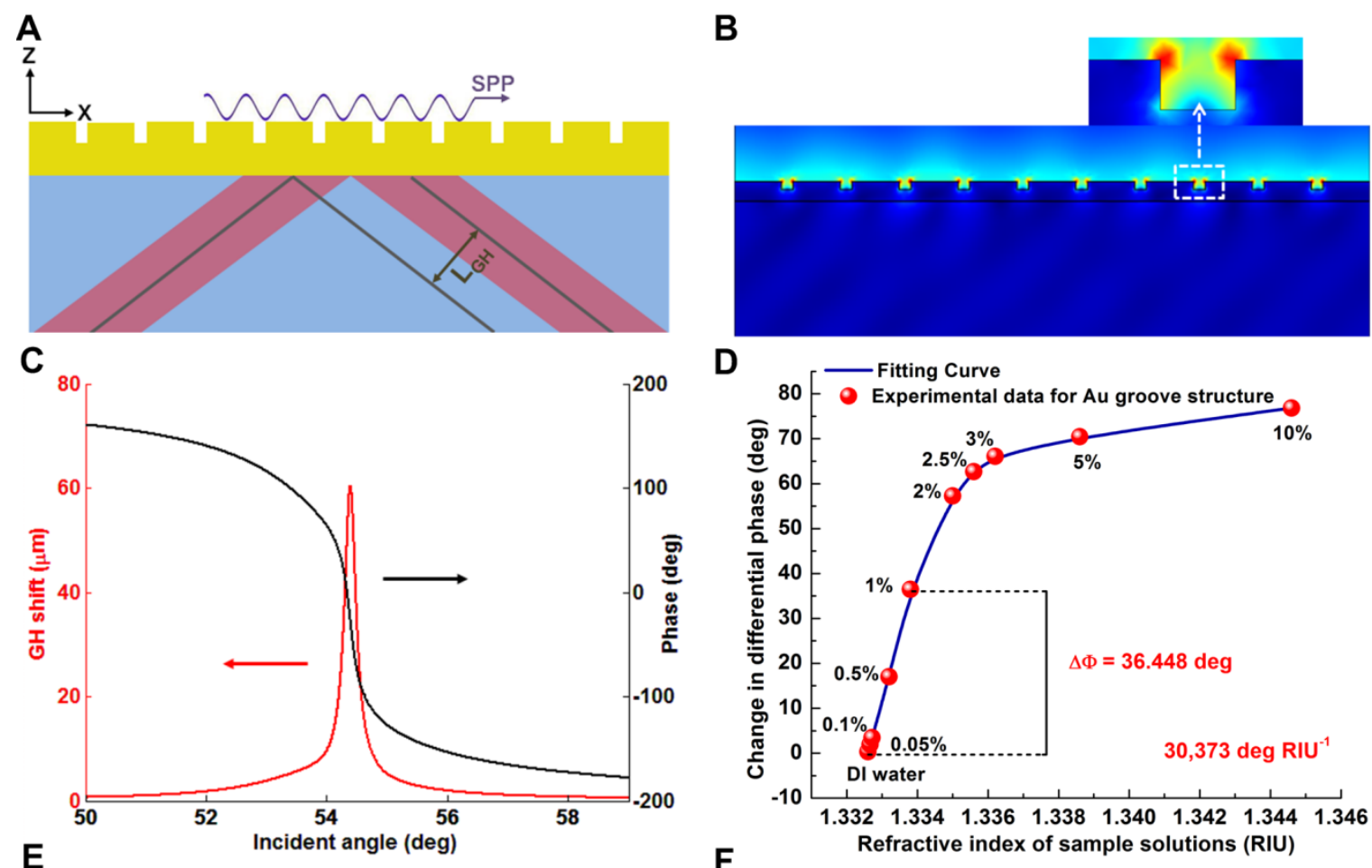

E Fitting curve
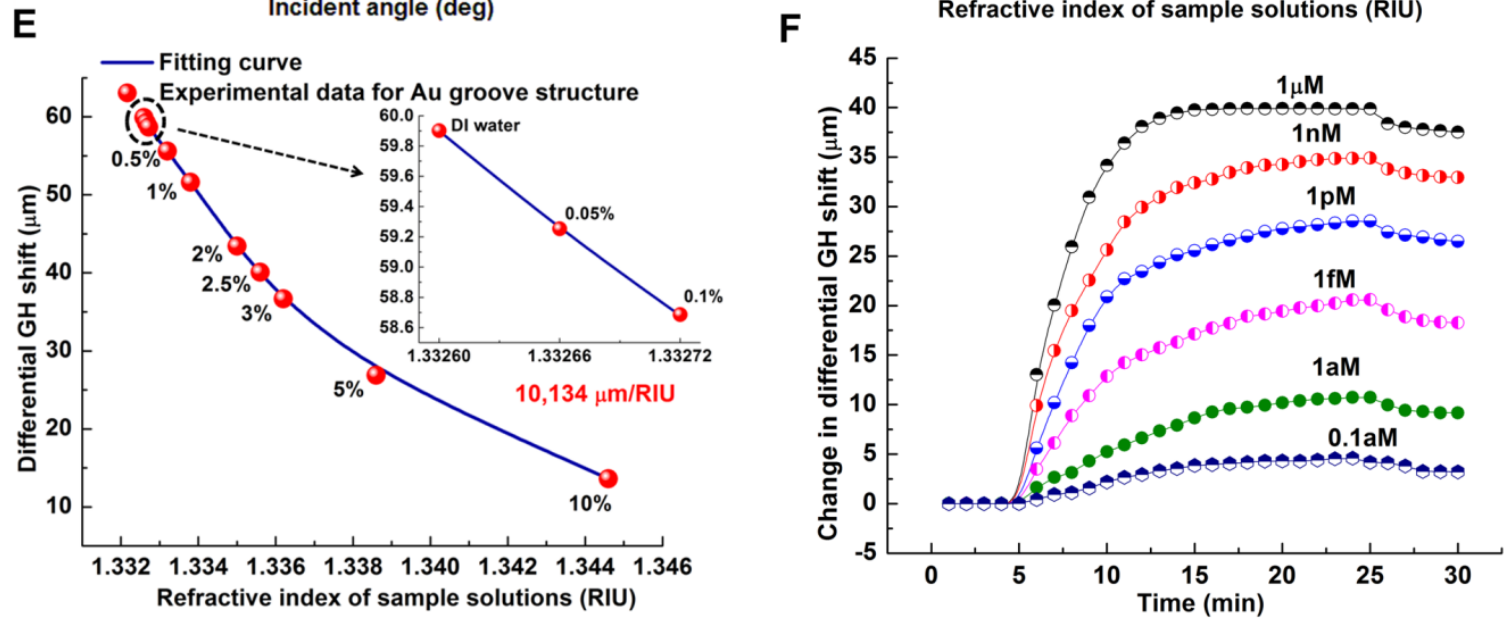

Figure 15: Phase-sensitive and Goos-Hänchen (GH)-sensitive plasmonic sensing platform based on the groove metasurface structures [97]. 


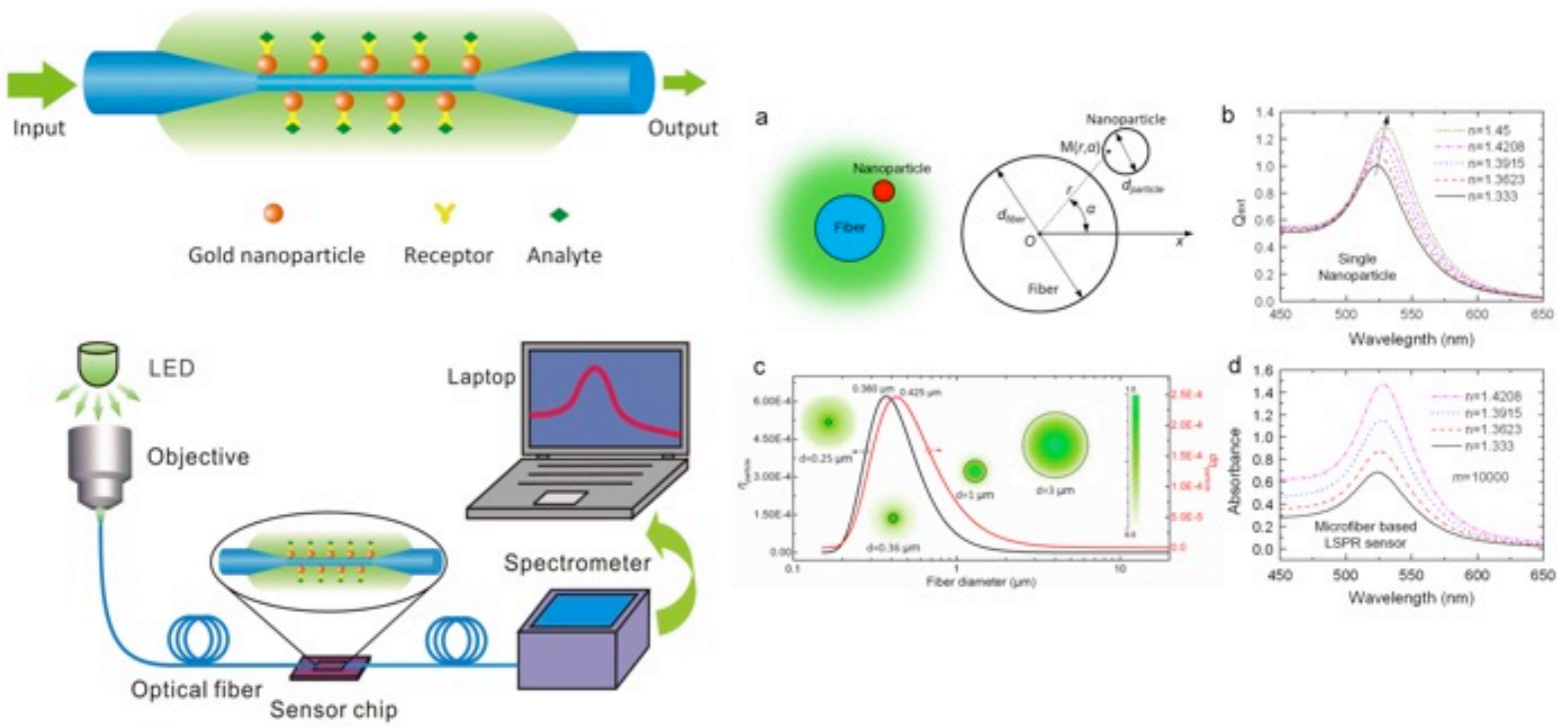

Figure 16: A schematic diagram showing the principle of optical micro/nanofiber (OMNF)based localized surface plasmon resonance (LSPR) sensor [98].

\section{References:}

[1] R.W. Wood, On a remarkable case of uneven distribution of light in a diffraction grating spectrum, Phil. Magm. 4 (1902) 396-402.

[2] A. Otto, "Excitation of nonradiative surface plasma waves in silver by the method of frustrated total reflection," Zeitschrift für Physik, vol. 216, pp. 398-410, 1968

[3] E. Kretschmann and H. Reather, "Radiative decay of non-radiative surface plasmons excited by light," Zeitschrift für Naturforschung, vol. 23, pp. 2135-2136, 1968

[4] J. Zenneck, "Uber die Fortpflanztmg ebener elektro-magnetischer Wellen langs einer ebenen Leiterflache und ihre Beziehung zur drahtlosen Telegraphie," Annals der Physik, vol. 23, pp. 846-866, 1907

[5] R. H. Ritchie, "Plasma losses by fast electrons in thin films," Phys. Rev., vol. 106, pp. 874 $881,1957$.

[6] C. J. Powell and J. B. Swan, "Effect of oxidation on the characteristic loss spectra of aluminum and magnesium," Phys. Rev., vol. 118, pp. 640-643, 1960.

[7] E. A. Stern and R. A. Ferrell, "Surface plasma oscillations of a degenerate electron gas," Phys. Rev., vol. 120, pp. 130-136, 1960.

[8] I Pockrand, J D Swalen, J G Gordon and M R Phllpott, Surface plasmon spectroscopy of organic monolayer assemblies, Surface Sczence, 74 (1977) 237 - 244

[9] G Wahling, Arachldate layers on Ag and Au substrates detected by the ATR method, Z Nuturforsch , 33a (1978) $536-539$

[10] C. Nylander, B. Liedberg, T. Lind, Gas detection by means of surface plasmons resonance, Sensors and Actuators 3 (1982) 79-88. 
[11] B. Liedberg, C. Nylander, I. Lundstrom, Surface plasmons resonance for gas detection and biosensing, Sensors and Actuators 4 (1983) 299-304.

[12] Lijie H, Quentin P, Iban L, Aritz Y. S., Amaia P, Amaia Z, Daniel M, Rabah B, Sabina S, Label-free femtomolar cancer biomarker detection in human serum using grapheme coated surface plasmon resonance chip, in press

[13] E. Mauriz, A. Calle, J. J. Manclus, A. Montoya, L. M. Lechuga, Multi-analyte SPR immunoassays for environmental biosensing of pesticides, Anal. Bioanal. Chem., 387 (2012) 1449-1458

[14] Li, Y., Liu, X. \& Lin, Z. Recent developments and applications of surface plasmon resonance biosensors for the detection of mycotoxins in foodstuffs. Food. Chem. 132 (2012) 1549-1554.

[15] M. Seo, J. Lee, M. Lee, Grating-coupled surface plasmon resonance on bulk stainless steel, OPTICS EXPRESS 25, 22 (2017) 26941

[16] Kort Bremer, Bernhard Roth, Fibre optic surface plasmon resonance sensor system designed for smartphones, OPTICS EXPRESS 23, (2015) 17179

[17] AK Sharma, R Jha, BD Gupta, Fiber-optic sensors based on surface plasmon resonance: a comprehensive review, IEEE Sensors Journal 20077 (8), 1118-1129

[18] S. Chandra. R. Bharadwaj, S. Mukherjia, Label free ultrasensitive optical sensor decorated with polyaniline nanofibers: Characterization and immunosensing application, Sens. Actuators B, 240 (2017), 443-450

[19] Hanna Dorozinska, Oleksandr Lyapin, Glib Dorozinsky and Volodymyr Maslov, Current Trends in Technology and Materials of Sensors Based on Surface Plasmon Resonance, Adv Material Sci Engg, 2 (2018)

[20] Qingling Ouyang, Shuwen Zeng, Li Jiang, Junle Qu, Xuan-Quyen Dinh, Jun Qian, Sailing He, Philippe Coquet, Ken-Tye Yong, Two-dimensional transition metal dichalcogenide enhanced phase-sensitive plasmonic biosensors: theoretical insight, The Journal of Physical Chemistry C 121 (11), 2017 6282-6289.

[21] L Jiang, S Zeng, Q Ouyang, XQ Dinh, P Coquet, J Qu, S He, KT Yong, GrapheneTMDC - graphene hybrid plasmonic metasurface for enhanced biosensing: a theoretical analysis, Physica Status Solidi A: Applications And Materials Science, (2017) 1-15.

[22] Gerardo A. Lopez, M.-Carmen Estevez, Maria Solera, Laura M. Lechuga, Recent advances in nanoplasmonic biosensors: applications and lab-on-a-chip integration, Nanophotonics 2017; 6(1): 123-136.

[23] J. Homola, Present and future of surface plasmon resonance biosensorm Anal. Bioanal Chem. (2003) $277: 528-539$.

[24] Ernande F. Melo, Eduardo Fontana, Optimization of Metal Gratings for SPR Sensing Applications, SBMO/IEEE MTT-S International Microwave and Optoelectronics Conference (IMOC 2011)

[25] Eduardo Fontana, Theoretical and experimental study of the surface plasmon resonance effect on a recordable compact disk,Optical Society of America, Vol. 43, No.1 (2004).

[26] D G Hong, T W Kim, K B Kim, J S Yuk, K S Ha, Development of an immunosensor with angular interrogation-based SPR spectroscopy, Meas. Sci. Technol. 18 (2007) 1367-1371 
[27] Zhe Zhang, Dan-feng Lu, Qian Liu, Zhi-mei Qi, Liangbao Yang, Jinhuai Liu, Wavelengthinterrogated surface plasmon resonance sensor with mesoporous-silica-film-enhanced sensitivity to small molecules, Analyst, 2012, 137, 4822

[28] Joong-Sung Lee, Trung Huynh, Su-Yong Lee, Kwang-Geol Lee, Jinhyoung Lee, Mark Tame, Carsten Rockstuhl, Changhyoup Lee, Quantum noise reduction in intensity-sensitive surface-plasmon-resonance sensors, PHYSICAL REVIEW A 96, 033833 (2017)

[29] Shijie Deng, Peng Wang, Xinglong Yu, Phase-Sensitive Surface Plasmon Resonance Sensors: Recent Progress and Future Prospects, Sensors 2017, 17, 2819

[30] Celina M. Miyazaki, Flávio M. Shimizu, Marystela Ferreira, 6 - Surface Plasmon Resonance (SPR) for Sensors and Biosensors, Nanocharacterization Techniques, Micro and Nano Technologies, 2017, 183-200.

[31] Ley Hood Hong, Asiah Yahaya, and Yusof Munajat, Simulation of surface plasmon resonance sensor, AIP Conference Proceedings 1674, 020015 (2015)

[32] Sandrine Filion-Côté, Philip J. R. Roche, Amir M. Foudeh, Maryam Tabrizian, and Andrew G. Kirk, Design and analysis of a spectro-angular surface plasmon resonance biosensor operating in the visible spectrum, Review of Scientific Instruments 85, 093107 (2014).

[33] J. Homola, Surface plasmon resonance sensors for detection of chemical and biological species, Chem. Rev. 108 (2008) 462-493.

[34] K. Yokoyama, M. Oishi, and M. Oshima, Development of an enhanced surface plasmon resonance sensor substrate by investigating a periodic nanohole array configuration, J. Appl. Phys. 118 (2015) 023101

[35] R. Boruah, D. Mohanta, A Choudhury, P. Nath, G. A. Ahmed, Surface plasmon resonancebased protein bio-sening using a Krestschmann configured double prism arrangement, IEEE sensors journal, 15 (2015) 6791-6796.

[36] Y. W. Fen, W. M. M. Yunus, Z. A. Talib, N. A. Yusof, Development of surface plasmon resonance sensor for determining zinc ion using novel active nanolayers as probe, Spectrochemica Acta Part A: Molecular and Biomolecular Spectroscopy, 134, (2015), 48-52.

[37] D. G. Hong, T. W. Kim, K. B. Kim, J. S. Yuk, K. S. Ha, Development of an immunosensor with angular interrogation-based SPR spectroscopy, Meas. Sci. echnol. 18 (2007) 1367-1371.

[38] Hye Rim Sim, Alastair W. Wark and Hye Jin Lee, Attomolar detection of protein biomarkers using biofunctionalized gold nanorods with surface plasmon resonance, Analyst 135 (2010) 2528-2532.

[39] Yi Wang, Wolfgang Knoll, Jakub Dostalek, Bacterial Pathogen Surface Plasmon Resonance Biosensor Advanced by Long Range Surface Plasmons and Magnetic Nanoparticle Assays, Anal. Chem., 84 (19) (2012) 8345-8350.

[40] Qiaoling Yu, Qihui Wang, Bianmiao Li, Qingyu Lin, Yixiang Duan, Technological Development of Antibody Immobilization for Optical Immunoassays: Progress and Prospects, Crit. Rev. Anal. Chem. (2015) 45, 62-75.

[41] A. Kausaite-Minkstimiene, A. Ramanaviciene, J. Kirlyte, A. Ramanavicius, Comparative Study of Random and Oriented Antibody Immobilization Techniques on the Binding Capacity of Immunosensor, Anal. Chem. 2010, 82, 6401-6408. 
[42] Nicholas G. Welch, Judith A. Scoble, Benjamin W. Muir, Paul J. Pigram, Orientation and characterization of immobilized antibodies for improved immunoassays (Review), Biointerphases 12, 02D301 (2017).

[43] J. M. Lee, H. K. Park, Y. Jung, J. K. Kim, S. O. Jung, B. H. Chung, Direct Immobilization of Protein G Variants with Various Numbers of Cysteine Residues on a Gold Surface Anal. Chem. 2007, 79, 2680-2687.

[44] Ji Eun Lee, Jeong Hyun Seo, Chang Sup Kim, Yunkyeoung Kwon, Jeong Hyub Ha, Suk Soon Choi, Hyung Joon Cha, A comparative study on antibody immobilization strategies onto solid surface, Korean J. Chem. Eng., 30(10), 1934-1938 (2013)

[45] Tomizaki, K.; Usui, K.; Mihara, H. ChemBioChem 2005, 6, 782- 799.

[46] Ma, X.-X.; Liu, H.-Z.; Tang, J.-J.; Guo, L.; Xie, J.-W. Comparison of Three EnzymeLinked Immunosorbent Assay Methods for Quantitative Determination of Ricin, Chin. J. Anal. Chem. 2011, 39, 685-689.

[47] Federica Rusmini, Zhiyuan Zhong, Jan Feijen, Protein Immobilization Strategies for Protein Biochips, Biomacromolecules 2007, 8, 1775-1789.

[48] 2J. L. Acero Sanchez, A. Fragoso, H. Joda, G. Suarez, C. J. McNeil, and C. K. O'Sullivan, Anal. Bioanal. Chem. 408, 5337 (2016).

[49] Uchida, K.; Otsuka, H.; Kaneko, M.; Kataoka, K.; Nagasaki, Y. Anal. Chem. 2005, 77, 1075-1080.

[50] C. O’Dwyer, G. Gay, B. Viaris de Lesegno, J. Weiner, The Nature of Alkanethiol SelfAssembled Monolayer Adsorption on Sputtered Gold Substrates, Langmuir 2004, 20, 81728182.

[51] Johanna Stettner, Adolf Winkler, Characterization of Alkanethiol Self-Assembled Monolayers on Gold by Thermal Desorption Spectroscopy, Langmuir 2010, 26(12), 9659-9665. [52] Czanderna, A. W.; King, D.; Spaulding, D. J. Vac. Sci. Technol., A 1991, 5, 2607

[53] Frey, B. L.; Corn, R. M. Anal. Chem. 1996, 68, 3187.

[54] Duevel, R. V.; Corn, R. M. Anal. Chem. 1992, 64, 337.

[55] V. Lebec, S. Boujday, C. Poleunis, C. M. Pradier, and A. Delcorte, J. Phys. Chem. C 118, 2085 (2014).

[56] S. N. Choudhury, B. Konwar, S. Kaur, R. Doley, B. Mondal, Study on Snake Venom Protein-Antibody Interaction by Surface Plasmon Resonance Spectroscopy, Photonic Sensors, 8, 3, 2018, 193-202

[57] Yam, C. M.; Deluge, M.; Tang, D.; Kumar, A.; Cai, C. J. Colloid Interface Sci. 2006, 296, $118-130$

[58] J. M. Goddard and J. H. Hotchkiss, Prog. Polym. Sci. 32, 698 (2007).

[59] Fernandez-Lafuente, R.; Rosell, C. M.; Rodriguez, V.; Santana, C.; Soler, G.; Bastida, A.; Guisan, G. M Enzyme Microb. Technol. 1993, 15, 546-550.

[60] Viitala, T.; Vikholm, I.; Peltonen, J. Langmuir 2000, 16, 4953-4961.

[61] A. K. Trilling, J. Beekwilder, and H. Zuilhof, Analyst 138, 1619 (2013).

[62] H. Sharma and R. Mutharasan, Anal. Chem. 85, 2472 (2013) 
[63] Qian, W.; Xu, B.; Yao, D.; Lin, Y.; Wu, L.; Wang, C.; Yu, F.; Lu, Z.; Wei, Y. Site-Directed Immobilization of Immunoglobulin $\mathrm{G}$ on 3- Aminopropyltriethoxylsilane Modified Silicon Wafer Surfaces, Mater. Sci. Eng. C 1999, 8, 475-480.

[64] James V. Staros, Rick W.Wright, Deborah M. Swingle, Enhancement by Nhydroxysulfosuccinimide of water-soluble carbodiimide-mediated coupling reactions, Anal. Biochem. 156, 1986 220-222.

[65] Thermo Scientific Crosslinking Technical Handbook.

[66] B. Konwar, Fabrication of Surface Plasmon Resonance biosensor for snake venom protein detection, Master's Thesis, (2017), Department of ECE, Tezpur University.

[67] S. Swarnakar, Fabrication of Surface plasmon resonance biochip to study interaction of IgG with monoclonal anti IgG, Master's Thesis, (2018), Department of ECE, Tezpur University.

[68] X. Zhao, Y. Cai, T. Wang, Y. Shi, G. Jiang, Preparation of alkanethiolate-functionalized core/shell $\mathrm{Fe}_{3} \mathrm{O}_{4} @ \mathrm{Au}$ nanoparticles and its interaction with several typical target molecules, Anal. Chem., 2008, 80, 9091-9096.

[69] B. M. Amoli, S. Gumfekar, A. Hu, Y. N. Zhou, and B. Zhao, "Thiocarboxylate functionalization of silver nanoparticles: effect of chain length on the electrical conductivity of nanoparticles and their polymer composites," Journal of Materials Chemistry, 2012, 22(37): 20048-20056.

[70] E. Goormaghtigh, J. M. Ruysschaert, and V. Raussens, "Evaluation of the information content in infrared spectra for protein secondary structure determination," Biophysical Journal, 2006, 90(8): 2946-2957

[71] S. Mukherji, Munshi Imran Hussain, T. Kundu and Deepali Chandratre, "Development of a Surface Plasmon Resonance based Biosensing System", in Micro and Smart Devices and Systems, ed. K.J. Vinoy et al., Springer India, 2014, pp 49-72.

[72] S. Chakraborty, Development of surface plasmon resonance based portable refractometer, Master's Thesis, (2018), Department of ECE, Tezpur University.

[73] M. Piliarik, M. Vala, I. Tichý, J. Homola Compact and low-cost biosensor based on novel approach to spectroscopy of surface plasmons, Biosensors and Bioelectronics, 24 (2009) 34303435.

[74] Fátima Fernández, Katerina Hegnerová, Marek Piliarik, Francisco Sanchez-Baezaa, Jirí Homola, M.-Pilar Marco, A label-free and portable multichannel surface plasmon resonanceimmunosensor for on-site analysis of antibiotics in milk samples, Biosensors and Bioelectronics 26 (2010) 1231-1238.

[75] Yun Liu, Shimeng Chen, Qiang Liu, Jean-François Masson, and Wei Peng, Compact multichannel surface plasmon resonance sensor for real-time multi-analyte bio-sensing, Optics Express 23, (2015) 20540-20548.

[76] Zeng, S.; Baillargeat, D.; Ho, H.-P.; Yong, K.-T. Nanomaterials Enhanced Surface Plasmon Resonance for Biological and Chemical Sensing Applications. Chem. Soc. Rev. 2014, 43, 3426-3452 
[77] Jang, Y. H.; Chung, K.; Quan, L. N.; Š packova`, B.; S' ıppova, H.; Moon, S.; Cho, W. J.; Shin, H.-Y.; Jang, Y. J.; Lee, J.-E. ConfigurationControlled Au Nanocluster Arrays on Inverse Micelle Nano-Patterns: Versatile Platforms for SERS and SPR Sensors. Nanoscale 2013, 5, 12261-12271.

[78] Chih-Tsung Yang, Lin Wu, Xiaohu Liu, Nhung Thi Tran, Ping Bai, Bo Liedberg, Yi Wang, and Benjamin Thierry, Exploiting Surface-Plasmon-Enhanced Light Scattering for the Design of Ultrasensitive Biosensing Modality, Anal. Chem. 2016, 88, 23, 11924-11930.

[79] Yong-Xiang Jiang, Bing-Hong Liu, Xiao-Song Zhu, Xiao-Li Tang, and Yi-Wei Shi, Longrange surface plasmon resonance sensor based on dielectric/silver coated hollow fiber with enhanced figure of merit, Optics Letters 40, $744-747$ (2015).

[80] Zhaoyang Chen, Xin Zhao, Chengyou Lin, Shujing Chen, Liang Yin, and Yingchun Ding, Figure of merit enhancement of surface plasmon resonance sensors using absentee layer, Applied Optics 55, 6832-6835 (2016)

[81] A. Shalabney and I. Abdulhalim, Figure-of-merit enhancement of surface plasmon resonance sensors in the spectral interrogation, Optics Letters 37, 1175-1177 (2012).

[82] Rajan Jha and Anuj K. Sharma, High-performance sensor based on surface plasmon resonance with chalcogenide prism and aluminum for detection in infrared, Optics Letters, 34, 749-751 (2009).

[83] Romain Guider, Davide Gandolfi, Tatevik Chalyan, Laura Pasquardini, Alina Samusenko, Cecilia Pederzolli, Georg Pucker, Lorenzo Pavesi, Sensitivity and Limit of Detection of biosensors based on ring resonators, Sensing and Bio-Sensing Research, 62015 99-102.

[84] Nelson, S. G.; Johnston, K. S. and Yee, S. S., "High sensitivity surface plasmon resonance sensor based on phase detection", Sensors and Actuators B: Chemical, 1996, 35(1-3): 187-191.

[85] Chen X. and Jiang K., "Effect of aging on optical properties of bimetallic sensor chips", Optical Society of America, 2010, 18(2):1105-1112.

[86] Zynio S. A, Samoylov A. V, Surovtseva E. R, Mirsky V. M, and Shirshov Y. M., "Bimetallic layers increase sensitivity of affinity sensors based on surface plasmon resonance," Sensors, 2002, 2(2):62-70.

[87] Yuan X-C, Ong B H, Tan Y G, Zhang DW, Irawan R and Tjin S C, "Sensitivity-stabilityoptimized surface plasmon resonance sensing with double metal layers", Journal of Optics A: Pure and Applied Optics, 2006, 8(11): 959-963.

[88] R. Kashyup, S. Chakraborty, S. Swarnikar, G. Humbert, S. Zeng, B. Mondal, FWHM and sensitivity study of bimetallic SPR sensor chip, accepted for 2019 IEEE International Conference on Electrical, Computer and Communication Technologies (IEEE ICECCT 2019), SVS College of Engineering, Coimbatore, Tamil Nadu, India during 20 - 22, February 2019.

[89] Zeng, S.; Hu, S. Y.; Xia, J.; Anderson, T.; Dinh, X. Q.; Meng, X. M.; Coquet, P.; Yong, K. T., Graphene-MoS2 hybrid nanostructures enhanced surface plasmon resonance biosensors. Sensors and Actuators B-Chemical 2015, 207, 801-810.

[90] Zeng, S.; Sreekanth, K. V.; Shang, J. Z.; Yu, T.; Chen, C. K.; Yin, F.; Baillargeat, D.; Coquet, P.; Ho, H. P.; Kabashin, A. V.; Yong, K. T., Graphene-Gold Metasurface Architectures for Ultrasensitive Plasmonic Biosensing. Advanced Materials 2015, 27 (40), 6163-6169. 
[91] Novoselov, K. S.; Mishchenko, A.; Carvalho, A.; Castro Neto, A. H., 2D materials and van der Waals heterostructures. Science 2016, 353 (6298).

[92] Azizi, A.; Eichfeld, S.; Geschwind, G.; Zhang, K.; Jiang, B.; Mukherjee, D.; Hossain, L.; Piasecki, A. F.; Kabius, B.; Robinson, J. A.; Alem, N., Freestanding van der Waals Heterostructures of Graphene and Transition Metal Dichalcogenides. ACS Nano 2015, 9 (5), 4882-4890.

[93] Ouyang, Q.; Zeng, S.; Jiang, L.; Hong, L.; Xu, G.; Dinh, X.-Q.; Qian, J.; He, S.; Qu, J.; Coquet, P.; Yong, K.-T., Sensitivity enhancement of transition metal dichalcogenides/silicon nanostructure-based surface plasmon resonance biosensor. Scientific Reports 2016, 6, 28190.

[94] Britnell, L.; Ribeiro, R. M.; Eckmann, A.; Jalil, R.; Belle, B. D.; Mishchenko, A.; Kim, Y. J.; Gorbachev, R. V.; Georgiou, T.; Morozov, S. V.; Grigorenko, A. N.; Geim, A. K.; Casiraghi, C.; Neto, A. H. C.; Novoselov, K. S., Strong Light-Matter Interactions in Heterostructures of Atomically Thin Films. Science 2013, 340 (6138), 1311.

[95] Bahauddin, S. M.; Robatjazi, H.; Thomann, I., Broadband absorption engineering to enhance light absorption in monolayer $\mathrm{MoS}_{2}$. ACS Photonics 2016, 3 (5), 853-862.

[96] Mishra, A. K.; Mishra, S. K.; Verma, R. K., Graphene and beyond graphene MoS2: a new window in surface-plasmon-resonance-based fiber optic sensing. The Journal of Physical Chemistry C, 2016, 120, 2893-2900.

[97] Jiang, L.; Zeng, S.; Xu, Z.; Ouyang, Q.; Zhang, D.-H.; Chong, P. H. J.; Coquet, P.; He, S.; Yong, K.-T., Multifunctional Hyperbolic Nanogroove Metasurface for Submolecular Detection. Small 2017, 13 (30), 1700600.

[98] Li, K.; Zhou, W.; Zeng, S., Optical micro/nanofiber-based localized surface plasmon resonance biosensors: Fiber diameter dependence. Sensors 2018, 18 (10). 